\begin{tabular}{|c|c|}
\hline Citation & $\begin{array}{l}\text { Florian Maurin, Laurens Coox, Francesco Greco, Elke Deckers, Claus } \\
\text { Claeys, Wim Desmet, (2016), } \\
\text { Title: Bloch theorem for isogeometric analysis of periodic problems } \\
\text { governed by high-order partial differential equations } \\
\text { Computer Methods in Applied Mechanics and Engineering, Volume 311, } \\
\text { Pages } 743-763\end{array}$ \\
\hline Archived version & $\begin{array}{l}\text { Author manuscript: the content is identical to the content of the published } \\
\text { paper, but without the final typesetting by the publisher }\end{array}$ \\
\hline Published version & 10.1016/j.cma.2016.09.015 \\
\hline Journal homepage & http://www.sciencedirect.com/science/journal/00457825 \\
\hline Author contact & $\begin{array}{l}\text { florian.maurin@kuleuven.be } \\
+32 \text { (0)16 } 372391\end{array}$ \\
\hline IR & https://lirias.kuleuven.be/handle/123456789/550857 \\
\hline
\end{tabular}

(article begins on next page) 


\title{
Bloch theorem for isogeometric analysis of periodic problems governed by high-order partial differential equations
}

\author{
Florian Maurin ${ }^{\mathrm{a}, \mathrm{b}, *}$, Laurens Coox ${ }^{\mathrm{a}, \mathrm{b}}$, Francesco Greco $^{\mathrm{a}, \mathrm{b}}$, Elke Deckers $^{\mathrm{a}, \mathrm{b}}$, \\ Claus Claeys ${ }^{\mathrm{a}, \mathrm{b}}$, Wim Desmet ${ }^{\mathrm{a}, \mathrm{b}}$ \\ ${ }^{a} K U$ Leuven, Department of Mechanical Engineering, Division PMA \\ Celestijnenlaan 300B - box 2420, B-3001 Leuven, Belgium. \\ ${ }^{b}$ Member of Flanders Make.
}

\begin{abstract}
With the emergence of isogeometric analysis (IGA), numerical rotation-free solutions to high-order partial differential equations (PDEs) are facilitated. Indeed, IGA allows the use of high-order-continuous basis functions through the domain. For periodic systems, the Bloch theorem is useful for analyzing wave propagation, restricting the analysis to a single unit cell and applying periodic boundary conditions. However, in the literature, boundary conditions are only accounting for $\mathcal{C}^{0}$-continuity, and we propose in the present paper to enforce the $\mathcal{G}^{1}$-continuity as well or to maintain the angle in case of unit cells meeting at kinks. While $\mathcal{G}^{1}$-continuity is enforced by maintaining the collinearity between adjacent control points, in the presence of kinks, variations of the field derived with respect to opposite in-plane edge normals are linked by the Rodrigues rotation matrix. This method applied at collocation points belonging to the unit cell edges can be viewed as a novel approach of coupling multi-patch geometries with kinks, avoiding the use of nonlinear multipliers or additional bending strip elements. Both transfer-matrix and indirect methods of the Bloch theorem are investigated, for respectively quasi-one and quasi-two-dimensional wave propagation in periodic structures discretized by NURBS-based IGA. Dispersion curves of a set of numerical problems treating isogeometric rotation-free extensible elastica (beams) and Kirchhoff-Love shells are then analyzed.
\end{abstract}

\footnotetext{
*Corresponding author: florian.maurin@kuleuven.be
} 
Keywords: Bloch theorem, NURBS-based isogeometric analysis, high-order partial differential equation, multi-patch coupling, rotation-free discretization, $\mathcal{G}^{1}$-continuity and kinks

\section{Introduction}

Wave propagation in infinite periodic systems is of interest in a large range of applications, such as acoustic metamaterials [1, 2] and non-destructive evaluation for structural health monitoring [3]. To this end, the Bloch theorem also sometimes called the Bloch-Floquet theorem can be used, reducing the analysis to a single unit cell on which periodic boundary conditions are applied [4-7].

While periodic boundary conditions in previous works only account for the $\mathcal{C}^{0}$-continuity, the consequences of its direct use in higher-order partial differential equation (PDE) is demonstrated in the following example. We consider an infinite straight uniform Euler-Bernoulli beam governed by the fourth-order PDE

$$
\frac{\partial^{2} w}{\partial t^{2}}+C_{B} \frac{\partial^{4} w}{\partial x^{4}}=0
$$

where $w(x, t)$ is the transversal displacement, $t$ and $x$ the time and space variables, and $C_{B}$ the sound speed of flexural waves [8]. Considering a finite period, Bloch theorem in its classical version can be used but requires an extra degree of freedom (dof), the rotation $\theta=\frac{\partial w}{\partial x}$, to reduce the order of the PDE. If instead Eq. (1) is solved directly without enforcing the higherorder-continuity at the boundaries, the investigated problem is different from the original one and presents periodic hinges (Fig. 1). While for the Euler-

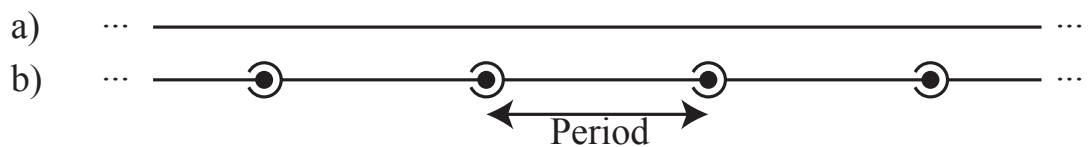

Figure 1: Infinite Euler-Bernoulli beam (a), and problem considered instead (b) if Eq. (1) is solved directly without accounting for the higher-order-continuity of the periodic boundary conditions.

Bernoulli beam problem, the second-order derivative of the transversal displacement corresponds to the beam rotation, high-order derivatives are not 
always physically meaningful quantities [9]. Moreover, with the emergence of isogeometric analysis (IGA), rotation-free formulations and in general higherorder PDEs become more and more popular.

Firstly introduced by Hughes et al. [10], IGA aims at filling the gap between computational mechanics and computer aided design (CAD) systems. It generalizes the finite element (FE) method by considering an isoparametric approach for which the basis functions used to represent the geometry are the same as the ones used to approximate the solution field. Since non-uniform rational B-splines (NURBS) are especially used in CAD systems, allowing the exact representation of conic geometries, NURBS-based IGA is considered here. One of its main advantages is that high-order continuous NURBS basis functions can be obtained, allowing direct solutions to high-order problems. Examples of high-order structural formulations for which NURBS-based IGA is used are the isogeometric rotation-free cable [11], extensible-elastica [9], Kirchhoff-Love rod [12], Bernoulli beam [13], and Kirchhoff-Love shell [14-17]. Additional application fields where high-order PDEs are involved are amongst others the gradient elasticity [18], the incompressible elasticity with stream functions [19], the Cahn-Hilliard phasefield model [20, 21], the Laplace-Beltrami triharmonic problem [21], and the Navier-Stokes equation [22].

Enforcing boundary conditions in the Bloch theorem can be viewed as multi-patch coupling. In NURBS-based IGA, $\mathcal{C}^{0}$-coupling is trivial for conforming patches [23], whereas it requires static condensation [24-27] or enrichment of the variational formulation (e.g. Nitsche [28], Lagrange multipliers [29, 30], and Penalty [31] methods) for non-conforming patches. For conforming patches and a uniform parametrization, enforcing the geometrical $\mathcal{G}^{1}$-continuity is equivalent to maintain the collinearity of adjacent control points at the joint interface $[14,18]$. Otherwise, if patches meet at a kink, for Kirchhoff-Love shells, the bending strip or the blended methods can be used [32,33]. An alternative consists in enforcing the rotational Dirichlet boundary conditions by the Penalty or Lagrange multiplier methods $[17,34,35]$. Note that for Reissner-Mindlin plates, the formulation is in general not rotation-free, and the $\mathcal{G}^{1}$-continuity or the kink angle can be maintained by using the director vectors [36, 37].

In the present work, we introduce a novel method for rotation-free discretizations with conforming patches meeting at kinks. The main idea is the introduction of the Rodrigues rotation matrix [38] linking the two derivatives computed with respect to the in-plane edge normals. 
The paper is organized as follows. In Sec. 2, NURBS-based IGA is briefly reviewed, and in Sec. 3, periodic boundary conditions are revisited. The inverse method [39-43] of the Bloch theorem is then presented in Sec. 3.4 for quasi-two-dimensional wave propagation. However, since this method is restricted to waves propagating without attenuation, the transfer-matrix approach $[5,44-46]$ is investigated as well for quasi-one-dimensional structures in Sec. 3.5. By quasi-one-dimensional wave propagation, we mean that the wave propagates in one direction whereas the structure can be up to three dimensions. Finally, in Sec. 4, the applicability of the proposed method is demonstrated in a set of numerical problems including wave propagation into a ring, a longitudinally-wrinkled helicoid, a flat and undulated shell, a periodic dome, and an expanded metal sheet. Both rotation-free extensible elastica (beam) and Kirchhoff-Love shell elements are used for these examples.

\section{Isogeometric Analysis}

\subsection{NURBS basis functions}

We consider the representation of the geometry by means of non-uniform rational B-splines (NURBS) [10]. A B-spline of polynomial order $p$ is defined by a knot vector $\Xi=\left\{\xi_{1} \leq \ldots \leq \xi_{n+p+1}\right\}$, with $n$ the number of basis functions forming the B-spline. The $i^{\text {th }}(i=1, \ldots, n)$ B-spline basis function $N_{i}^{p}(\xi)$ is derived from the knot-vector using the Cox-De Boor recursive formula [47]:

$$
\begin{aligned}
& N_{i}^{0}(\xi)=\left\{\begin{array}{l}
1 \text { if } \xi_{i} \leq \xi<\xi_{i+1}, \\
0 \quad \text { otherwise, }
\end{array}\right. \\
& N_{i}^{p}(\xi)=\frac{\xi-\xi_{i}}{\xi_{i+p}-\xi_{i}} N_{i}^{p-1}(\xi)+\frac{\xi_{i+p+1}-\xi}{\xi_{i+p+1}-\xi_{i+1}} N_{i+1}^{p-1}(\xi), \quad \text { for } p \geq 1,
\end{aligned}
$$

and examples of B-spline basis functions with their first derivatives are shown in Fig. 2. Assigning a weight $w_{i} \in \mathbb{R}$ to every B-spline basis function, NURBS basis functions are defined by the ratio

$$
R_{i}^{p}(\xi)=\frac{N_{i}^{p}(\xi) w_{i}}{\sum_{i_{w}=1}^{n} N_{i_{w}}^{p}(\xi) w_{i_{w}}}
$$



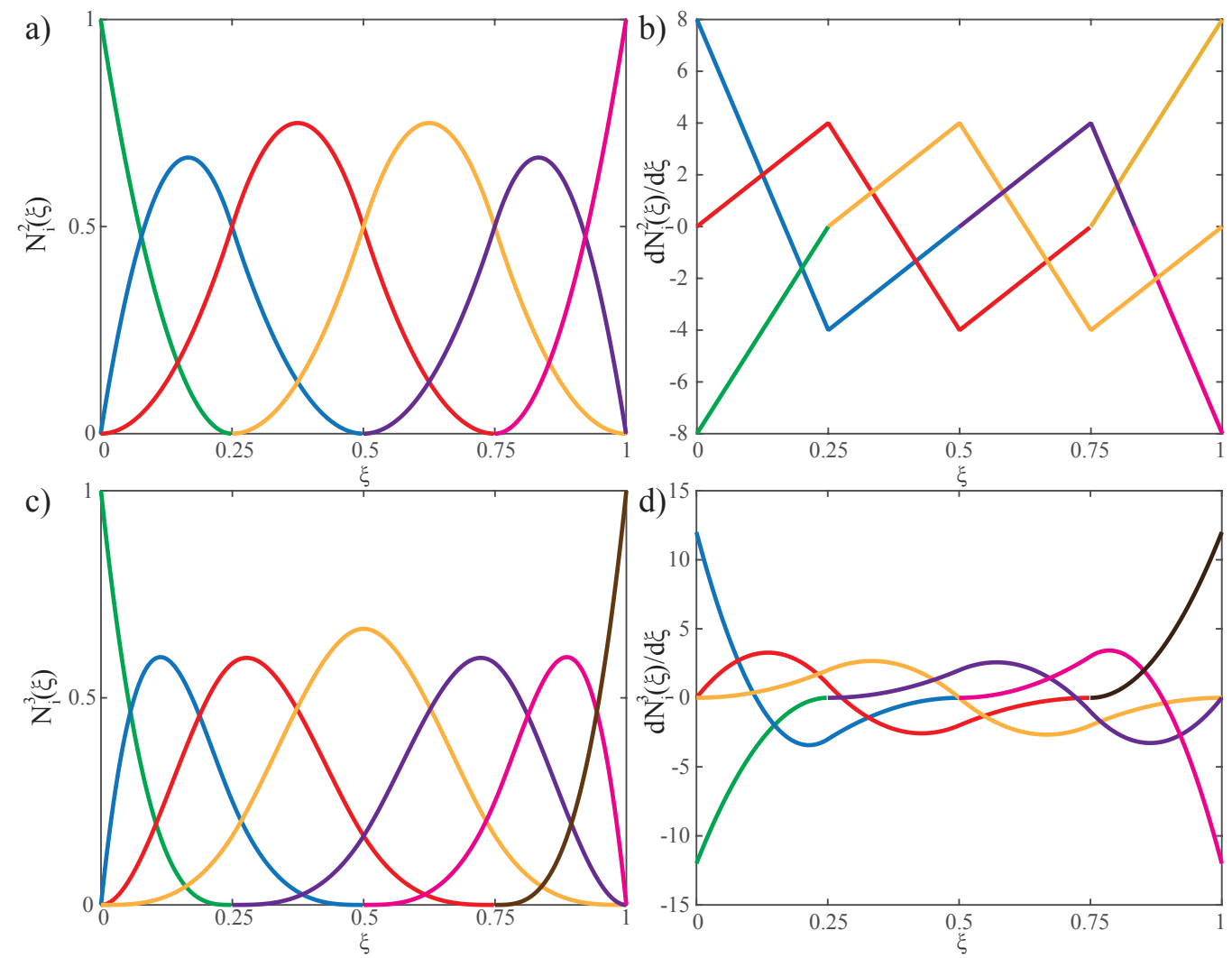

Figure 2: B-spline basis functions (Eq. (2)) (a,c) and its first derivatives (b,d) of order $p=2(\mathrm{a}, \mathrm{b})$ and $p=3(\mathrm{c}, \mathrm{d})$, and with the knot span $\Xi=\left\{\begin{array}{llllllllll}0 & 0 & 0 & 0.25 & 0.5 & 0.75 & 1 & 1 & 1\end{array}\right\}$ (a,b)

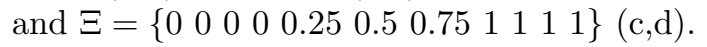

Given two univariate B-spline basis $N_{i}^{p}(\xi)(i=1, \ldots, n)$ and $M_{j}^{q}(\eta)(j=$ $1, \ldots, m)$, respectively of order $p$ and $q$, and associated to the two knot vectors $\Xi=\left\{\xi_{1} \leq \ldots \leq \xi_{n+p+1}\right\}$ and $\mathcal{H}=\left\{\eta_{1} \leq \ldots \leq \eta_{m+q+1}\right\}$, a bivariate NURBS basis function is defined as

$$
R_{i, j}^{p, k}(\xi, \eta)=\frac{N_{i}^{p}(\xi) M_{j}^{q}(\eta) w_{i, j}}{\sum_{i_{w}=1}^{n} \sum_{j_{w}=1}^{m} N_{i_{w}}^{p}(\xi) M_{j_{w}}^{q}(\eta) w_{i_{w}, j_{w}}} .
$$

NURBS surfaces $\boldsymbol{S}(\xi, \eta)$ are defined by

$$
\boldsymbol{S}(\xi, \eta)=\sum_{i=1}^{n} \sum_{j=1}^{m} R_{i, j}^{p, k}(\xi, \eta) \mathbf{B}_{i, j},
$$


where $\mathbf{B}_{i, j}$ are the control points. NURBS bases allow exact representation of conical geometries. However, if all the weights are equal, NURBS degenerate to B-splines since B-spline basis functions form a partition of unity. Using the isogeometric approach, the displacement field $\boldsymbol{u}(\xi, \eta)$ is approximated by the same basis functions that represent the geometry such that

$$
\boldsymbol{u}(\xi, \eta)=\sum_{i=1}^{n} \sum_{j=1}^{m} R_{i, j}^{p, k}(\xi, \eta) \mathbf{u}_{i, j}
$$

where $\mathbf{u}_{i, j}$ is the displacement at the control point $\mathbf{B}_{i, j}$.

Knot vectors define a partition of the parametric domain and non-uniform knot vectors and repeated knots are the key of the NURBS flexibility, allowing locally-refined, geometric descriptions and reduced continuity of the basis functions. In particular, across the knot $\xi_{i}$ of multiplicity $m_{i}$ such that

$1 \leq m_{i} \leq p$, the basis functions are $\mathcal{C}^{p-m_{i}}$-continuous. For the purpose of the present work, at least globally $\mathcal{C}^{p-m_{i}}$ basis functions are used for internal knots with $1 \leq m_{i} \leq p-1$ for $p \geq 2$. Moreover, if the multiplicity of the first and last knot is $p+1$, the knot vector is open (or clamped), whereas if this multiplicity is smaller than $p+1$, the knot vector is unclamped.

\subsection{Equation of motion}

In the present work, we focus on periodic problems governed by high-order PDEs. While the formulations are not explicitly detailed here since they depend on the considered PDE, we assume that the discretization is rotationfree and performed with the Galerkin method, such that symmetric mass (M) and stiffness (K) matrices can be obtained. Since these quantities are nonlinear for large deformations, the deformation around the equilibrium position is assumed to be small to allow their linearization. Assuming harmonic waves with angular frequency $\omega$, the equation of motion for the discretized periodic unit cell reads

$$
\left(\mathbf{K}-\omega^{2} \mathbf{M}\right) \mathbf{u}=\mathbf{f},
$$

where $\mathbf{u}$ and $\mathbf{f}$ are are displacement and force vectors corresponding to the control variables, respectively.

\section{Bloch Theorem For High-Order Partial Differential Equations}

Wave propagation in quasi-two-dimensional periodic structures is investigated with the Bloch theorem, restricting the analysis to a single unit cell, 
and taking advantage of periodic boundary conditions [4]. We denote $\boldsymbol{r}_{P}$ the position of the point $P$ within the reference cell, and by $\boldsymbol{\rho}_{P}=\boldsymbol{r}_{P}+n_{x} \boldsymbol{e}_{x}+n_{y} \boldsymbol{e}_{y}$ the same position $P$ relative to the $\left\{n_{x} n_{y}\right\}$-th unit cell, where $\boldsymbol{e}_{x}$ and $\boldsymbol{e}_{y}$ are the basis vectors of the direct lattice. Assuming a harmonic wave with angular frequency $\omega$ and amplitude $\hat{\boldsymbol{u}}$, the wave displacement $\boldsymbol{u}\left(\boldsymbol{r}_{P}, t\right)$ at time $t$ and position $\boldsymbol{r}_{P}$ is given by

$$
\boldsymbol{u}\left(\boldsymbol{r}_{P}, t\right)=\hat{\boldsymbol{u}} e^{\boldsymbol{k} \cdot \boldsymbol{r}_{P}-\mathrm{i} \omega t},
$$

where $\mathrm{i}^{2}=-1$ and $\boldsymbol{k}$ is the wave vector. The wave at the point $\boldsymbol{\rho}_{P}$ yields

$$
\boldsymbol{u}\left(\boldsymbol{\rho}_{P}, t\right)=\boldsymbol{u}\left(\boldsymbol{r}_{P}, t\right) e^{\left(n_{x} \mu_{x}+n_{y} \mu_{y}\right)},
$$

where the propagation constant components $\mu_{x}=\boldsymbol{k} \cdot \boldsymbol{e}_{x}$ and $\mu_{y}=\boldsymbol{k} \cdot \boldsymbol{e}_{y}$ are complex numbers such that their real and imaginary parts represent the attenuation and phase constants, respectively.

Given two open knot vectors such that $\Xi=\left\{\xi_{1}=0 \leq \ldots \leq \xi_{n+p+1}=\right.$ $1\}$ and $\mathcal{H}=\left\{\eta_{1}=0 \leq \ldots \leq \eta_{m+q+1}=1\right\}$, for problems requiring $\mathcal{C}^{0}$ continuity, the relation between opposite sides of the unit cell are derived for the displacement field $\boldsymbol{u}(\xi, \eta)$ from Eq. (9) and from equilibrium conditions for the force field $\boldsymbol{f}(\xi, \eta)[6]$ such that:

$$
\begin{aligned}
& \boldsymbol{u}(1, \eta)=+e^{\mu_{x}} \boldsymbol{u}(0, \eta), \\
& \boldsymbol{u}(\xi, 1)=+e^{\mu_{y}} \boldsymbol{u}(\xi, 0), \\
& \boldsymbol{f}(1, \eta)=-e^{\mu_{x}} \boldsymbol{f}(0, \eta), \\
& \boldsymbol{f}(\xi, 1)=-e^{\mu_{y}} \boldsymbol{f}(\xi, 0) .
\end{aligned}
$$

For high-order PDEs, this relation has to be completed to account for the high-order continuity, and we focus first to the case of a smooth transition between two adjacent unit cells (illustrated in Fig. 3a for the case of a curve), whereas the case of units meeting at kinks (Fig. 3b) is investigated in Sec. 3.6.

\section{1. $\mathcal{G}^{1}$-continuity between two adjacent unit cells}

For problems requiring $\mathcal{G}^{1}$-continuity between two adjacent unit cells, the first derivatives in the parametric space of opposite sides are linked by the 

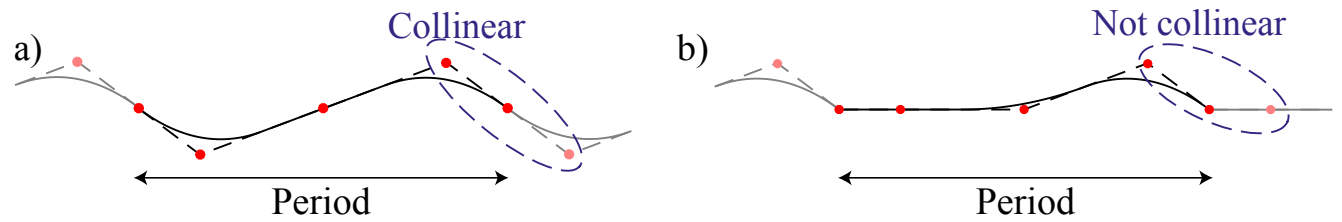

Figure 3: Periodic NURBS curve with control points represented by $\bullet$. $\mathcal{G}^{1}$-continuity (a) and kink (b) between two adjacent unit cells.

following relations:

$$
\begin{aligned}
& \frac{\partial \boldsymbol{u}(1, \eta)}{\partial \xi}=+e^{\mu_{x}} c_{0}(\eta) \frac{\partial \boldsymbol{u}(0, \eta)}{\partial \xi}, \\
& \frac{\partial \boldsymbol{u}(\xi, 1)}{\partial \eta}=+e^{\mu_{y}} c_{0}(\xi) \frac{\partial \boldsymbol{u}(\xi, 0)}{\partial \eta} \\
& \frac{\partial \boldsymbol{f}(1, \eta)}{\partial \xi}=-e^{\mu_{x}} c_{0}(\eta) \frac{\partial \boldsymbol{f}(0, \eta)}{\partial \xi} \\
& \frac{\partial \boldsymbol{f}(\xi, 1)}{\partial \eta}=-e^{\mu_{y}} c_{0}(\xi) \frac{\partial \boldsymbol{f}(\xi, 0)}{\partial \eta}
\end{aligned}
$$

where the geometrical constants $c_{0}$ are defined in terms of the original geometry $\boldsymbol{S}$ by

$$
\begin{aligned}
& c_{0}(\eta)=\left\|\frac{\partial \boldsymbol{S}(1, \eta)}{\partial \xi}\right\| /\left\|\frac{\partial \boldsymbol{S}(0, \eta)}{\partial \xi}\right\|, \\
& c_{0}(\xi)=\left\|\frac{\partial \boldsymbol{S}(\xi, 1)}{\partial \eta}\right\| /\left\|\frac{\partial \boldsymbol{S}(\xi, 0)}{\partial \eta}\right\| .
\end{aligned}
$$

Note that tangential to the interface, the $\mathcal{G}^{1}$-continuity is automatically ensured by the $\mathcal{C}^{0}$ one (e.g. $\left.\frac{\partial \boldsymbol{u}(1, \eta)}{\partial \eta}=e^{\mu_{x}} c_{0}(\eta) \frac{\partial \boldsymbol{u}(0, \eta)}{\partial \eta}\right)$. Before considering wave propagation in multiple directions, we focus first on the quasi-onedimensional case.

\subsection{Quasi-one-dimensional wave propagation}

The dofs are reorganized into left, right, and internal regions, denoted respectively ()$_{L},()_{R}$, and ()$_{I}$ (Fig. 4a,b), such that the generalized dis-

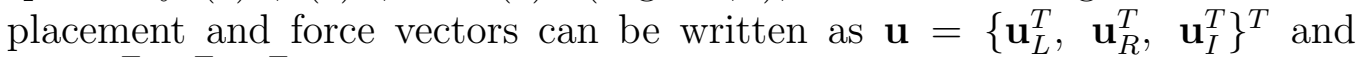
$\mathbf{f}=\left\{\mathbf{f}_{L}^{T}, \mathbf{f}_{R}^{T}, \mathbf{f}_{I}^{T}\right\}^{T}$. 
a)

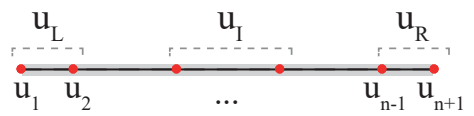

b)

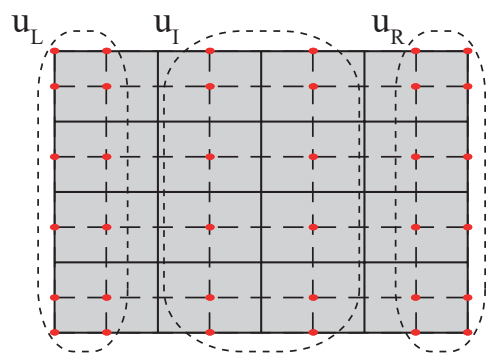

c)

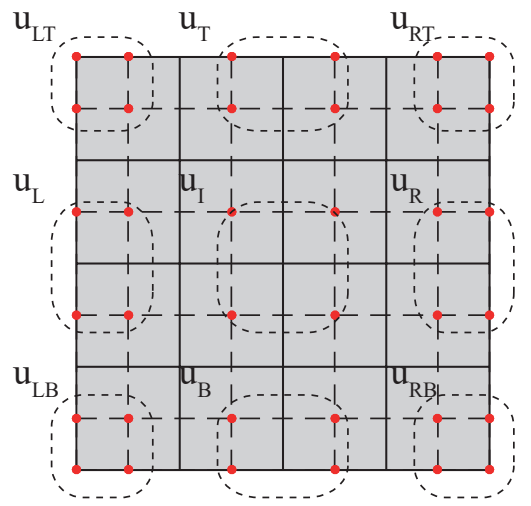

Figure 4: Examples of quasi-one-dimensional $(a, b)$ and quasi-two-dimensional (c) wave propagation into structures constructed from univariate (a) and bivariate (b,c) NURBS basis.

Considering a curvilinear structure constructed from a univariate NURBS basis $R_{i}^{p}(\xi)$ (Fig. 4a), using Eq. (6), the displacement and first derivative at one extremity are supported by one and two basis functions, respectively (Eq. (2) and Fig. 2). Denoting $\mathbf{u}_{i}$ the displacements of the $i^{\text {th }}$ control point $(i=1, \ldots, n$ from left to right), left, right and internal regions are composed by $\mathbf{u}_{L}=\left\{\mathbf{u}_{1}^{T}, \mathbf{u}_{2}^{T}\right\}^{T}, \mathbf{u}_{R}=\left\{\mathbf{u}_{n-1}^{T}, \mathbf{u}_{n}^{T}\right\}^{T}$, and $\mathbf{u}_{I}=\left\{\mathbf{u}_{3}^{T}, \cdots, \mathbf{u}_{n-2}^{T}\right\}^{T}$ (Fig. 4a). Eqs. (10a) and (11a) can be recasted in terms of the control variables for displacements

$$
\begin{aligned}
& {\left[\begin{array}{c}
\boldsymbol{u}(0) \\
\frac{d \boldsymbol{u}(0)}{d \xi}
\end{array}\right]=\left[\left[\begin{array}{cc}
1 & 0 \\
\frac{d R_{1}^{p}(0)}{d \xi} & \frac{d R_{2}^{p}(0)}{d \xi}
\end{array}\right] \otimes \mathbf{I}_{d}\right]\left[\begin{array}{l}
\mathbf{u}_{1} \\
\mathbf{u}_{2}
\end{array}\right]} \\
& =\left[\mathbf{N}_{L} \otimes \mathbf{I}_{d}\right] \mathbf{u}_{L}, \\
& {\left[\begin{array}{c}
\boldsymbol{u}(1) \\
\frac{d \boldsymbol{u}(1)}{d \xi}
\end{array}\right]=\left[\left[\begin{array}{cc}
0 & 1 \\
\frac{d R_{n-1}^{p}(1)}{d \xi} & \frac{d R_{n}^{p}(1)}{d \xi}
\end{array}\right] \otimes \mathbf{I}_{d}\right]\left[\begin{array}{c}
\mathbf{u}_{n-1} \\
\mathbf{u}_{n}
\end{array}\right]} \\
& =\left[\mathbf{N}_{R} \otimes \mathbf{I}_{d}\right] \mathbf{u}_{R},
\end{aligned}
$$

where $\otimes$ is the Kronecker product and $\mathbf{I}_{d}$ an identity matrix whose size corresponds to $d$, the number of dof per control point. Proceeding similarly with the force vector, and then plugging Eqs. (13) and (14) into Eqs. (10) and (11) leads to a relation between the displacements/forces of opposite 
sides:

$$
\begin{aligned}
& \mathbf{u}_{R}=+e^{\mu_{x}} \mathbf{N}_{\mathrm{X}} \mathbf{u}_{L}, \\
& \mathbf{f}_{R}=-e^{\mu_{x}} \mathbf{N}_{\mathrm{X}} \mathbf{f}_{L},
\end{aligned}
$$

where

$$
\begin{aligned}
\mathbf{N}_{\mathbf{X}} & =\left[\mathbf{N}_{R} \otimes \mathbf{I}_{d}\right]^{-1}\left[\mathbf{N}_{L} \otimes \mathbf{I}_{d}\right] \\
& =\left[\mathbf{N}_{R}^{-1} \mathbf{N}_{L}\right] \otimes \mathbf{I}_{d} \\
& =\left[\begin{array}{cc}
1+c_{x}^{\prime} & -c_{x}^{\prime} \\
1 & 0
\end{array}\right] \otimes \mathbf{I}_{d} .
\end{aligned}
$$

The geometrical constant $c_{x}^{\prime}$ can be directly determined from the initial geometry $\boldsymbol{S}$ using the relation $\boldsymbol{S}_{R} \mathbf{N}_{\mathrm{X}}=\boldsymbol{S}_{L}$.

Similarly, for quasi-one-dimensional wave propagation in structures constructed from a bivariate NURBS base (Fig. 4b) and with collinear adjacent control points at the interface,

$$
\mathbf{N}_{\mathrm{X}}=\left[\begin{array}{ccccc}
1+c_{x, 1}^{\prime} & -c_{x, 1}^{\prime} & \cdots & 0 & 0 \\
1 & 0 & \cdots & 0 & 0 \\
\vdots & \vdots & \ddots & \vdots & \vdots \\
0 & 0 & \cdots & 1+c_{x, m}^{\prime} & -c_{x, m}^{\prime} \\
0 & 0 & \cdots & 1 & 0
\end{array}\right] \otimes \mathbf{I}_{d}
$$

\subsection{Quasi-two-dimensional wave propagation}

As processed in the one-dimensional case, the dofs are reorganized into left, right, bottom, top, left-bottom, left-top, right-bottom, right-top, and internal regions, denoted respectively ()$_{L},()_{R},()_{B},()_{T},()_{L B},()_{L T},()_{R B},()_{R T}$, and ( $)_{I}$ (Fig. 4c), such that the generalized displacement and force vectors can be written in the form $\mathbf{u}=\left\{\mathbf{u}_{L}^{T}, \mathbf{u}_{R}^{T}, \mathbf{u}_{B}^{T}, \mathbf{u}_{T}^{T}, \mathbf{u}_{L B}^{T}, \mathbf{u}_{L T}^{T}, \mathbf{u}_{R B}^{T}, \mathbf{u}_{R T}^{T}, \mathbf{u}_{I}^{T}\right\}^{T}$ and $\mathbf{f}=\left\{\mathbf{f}_{L}^{T}, \mathbf{f}_{R}^{T}, \mathbf{f}_{B}^{T}, \mathbf{f}_{T}^{T}, \mathbf{f}_{L B}^{T}, \mathbf{f}_{L T}^{T}, \mathbf{f}_{R B}^{T}, \mathbf{f}_{R T}^{T}, \mathbf{f}_{I}^{T}\right\}^{T}$. The matrix $\mathbf{N}_{\mathrm{X}}$ of Eq. (17) can be also reorganized into subgroups such that Eq. (15a) reads

$$
\left[\begin{array}{c}
\mathbf{u}_{R} \\
\mathbf{u}_{R B} \\
\mathbf{u}_{R T}
\end{array}\right]=e^{\mu_{x}}\left[\begin{array}{ccc}
\mathbf{N}_{\mathrm{XI} I} & \mathbf{0} & \mathbf{0} \\
\mathbf{0} & \mathbf{N}_{\mathbf{X} B B} & \mathbf{0} \\
\mathbf{0} & \mathbf{0} & \mathbf{N}_{\mathbf{X T} T}
\end{array}\right]\left[\begin{array}{c}
\mathbf{u}_{L} \\
\mathbf{u}_{L B} \\
\mathbf{u}_{L T}
\end{array}\right]
$$

For the wave propagating in the $y$ direction, a similar equation is obtained, such that

$$
\left[\begin{array}{c}
\mathbf{u}_{T} \\
\mathbf{u}_{L T} \\
\mathbf{u}_{R T}
\end{array}\right]=e^{\mu_{y}}\left[\begin{array}{ccc}
\mathbf{N}_{\mathrm{Y} I I} & \mathbf{0} & \mathbf{0} \\
\mathbf{0} & \mathbf{N}_{\mathrm{Y} L L} & \mathbf{0} \\
\mathbf{0} & \mathbf{0} & \mathbf{N}_{\mathrm{Y} R R}
\end{array}\right]\left[\begin{array}{c}
\mathbf{u}_{B} \\
\mathbf{u}_{L B} \\
\mathbf{u}_{R B}
\end{array}\right]
$$


where

$$
\mathbf{N}_{\mathrm{Y}}=\left[\begin{array}{cccccc}
1+c_{y, 1}^{\prime} & \cdots & 0 & -c_{y, 1}^{\prime} & \cdots & 0 \\
\vdots & \ddots & \vdots & \vdots & \ddots & \vdots \\
0 & \cdots & 1+c_{y, n}^{\prime} & 0 & \cdots & -c_{y, n}^{\prime} \\
1 & \cdots & 0 & 0 & \cdots & 0 \\
\vdots & \ddots & \vdots & \vdots & \ddots & \vdots \\
0 & \cdots & 1 & 0 & \cdots & 0
\end{array}\right] \otimes \mathbf{I}_{d}
$$

\subsection{Inverse method of the Bloch theorem}

By solving the systems (18) and (19), u can be expressed in terms of a reduced vector $\mathbf{u}^{(r)}$ as:

$\mathbf{u}=\left[\begin{array}{c}\mathbf{u}_{L} \\ \mathbf{u}_{R} \\ \mathbf{u}_{B} \\ \mathbf{u}_{T} \\ \mathbf{u}_{L B} \\ \mathbf{u}_{L T} \\ \mathbf{u}_{R B} \\ \mathbf{u}_{R T} \\ \mathbf{u}_{I}\end{array}\right]=\left[\begin{array}{cccc}\mathbf{I} & \mathbf{0} & \mathbf{0} & \mathbf{0} \\ e^{\mu_{x}} \mathbf{N}_{\mathrm{X} I I} & \mathbf{0} & \mathbf{0} & \mathbf{0} \\ \mathbf{0} & \mathbf{I} & \mathbf{0} & \mathbf{0} \\ \mathbf{0} & e^{\mu_{y}} \mathbf{N}_{\mathrm{Y} I I} & \mathbf{0} & \mathbf{0} \\ \mathbf{0} & \mathbf{0} & \mathbf{I} & \mathbf{0} \\ \mathbf{0} & \mathbf{0} & e^{\mu_{x}} \mathbf{N}_{\mathrm{X} B B} & \mathbf{0} \\ \mathbf{0} & \mathbf{0} & e^{\mu_{y}} \mathbf{N}_{\mathbf{Y} L L} & \mathbf{0} \\ \mathbf{0} & \mathbf{0} & e^{\mu_{x}+\mu_{y}} \mathbf{N}_{\mathbf{X} T T} \mathbf{N}_{\mathrm{Y} L L} & \mathbf{0} \\ \mathbf{0} & \mathbf{0} & \mathbf{0} & \mathbf{I}\end{array}\right]\left[\begin{array}{c}\mathbf{u}_{L} \\ \mathbf{u}_{B} \\ \mathbf{u}_{L B} \\ \mathbf{u}_{I}\end{array}\right]=\mathbf{A}(\boldsymbol{\mu}) \mathbf{u}^{(r)}$,

where $\mathbf{I}$ is the identity matrix and where $\mathbf{N}_{\mathrm{X} T T} \mathbf{N}_{\mathrm{Y} L L}$ can be replaced by $\mathbf{N}_{\mathrm{Y} R R} \mathbf{N}_{\mathrm{X} B B}$ since only 5 equations are independent among the 6 equations composing the systems (18) and (19). This is similarly to the coupling of four surface patches [48].

Defining the reduced force vector as $\mathbf{f}^{(r)}=\left\{\mathbf{f}_{L}^{T}, \mathbf{f}_{B}^{T}, \mathbf{f}_{L B}^{T}, \mathbf{f}_{I}^{T}\right\}^{T}$ and following the same procedure as the one used for displacements, Eqs. (10b) and (11b) lead to the relation

$$
\mathbf{f}=\mathbf{A}(\boldsymbol{\mu}+\pi) \mathbf{f}^{(r)} .
$$

Assuming no internal forces $\left(\mathbf{f}_{I}=\mathbf{0}\right)$, a purely propagating wave $(\operatorname{Re}(\boldsymbol{\mu})=$ 0), and substituting Eq. (21) into Eq. (7), then multiplying both sides by $\mathbf{A}^{H}(\boldsymbol{\mu})$, where $H$ denotes the conjugate transpose, gives:

$$
\left[\mathbf{K}^{(r)}(\boldsymbol{\mu})-\omega^{2} \mathbf{M}^{(r)}(\boldsymbol{\mu})\right] \mathbf{u}^{(r)}=\mathbf{0}
$$


where $\mathbf{K}^{(r)}(\boldsymbol{\mu})=\mathbf{A}^{H}(\boldsymbol{\mu}) \mathbf{K A}(\boldsymbol{\mu})$ and $\mathbf{M}^{(r)}(\boldsymbol{\mu})=\mathbf{A}^{H}(\boldsymbol{\mu}) \mathbf{M A}(\boldsymbol{\mu})$ are the reduced (or condensed) stiffness and mass matrix, respectively. Eq. (23) is an eigenvalue problem which gives eigenvalues $\omega$ corresponding to the propagation constants $\boldsymbol{\mu}$. However, by assuming a wavenumber purely imaginary, the attenuation part of the dispersion relation cannot be obtained with this method, contrary to the transfer-matrix approach.

\subsection{Direct method of the Bloch theorem (transfer-matrix approach)}

Instead of fixing the wavenumber and looking for associated frequencies, one can fix the frequency and compute the propagation constants. While this method has been recently extended to two-dimensional wave propagation $[45,46,49]$, we focus here on quasi-one-dimensional problems [44] for waves propagating in the $x$ direction.

As done for the force and displacement vectors, the dynamic stiffness matrix (Eq. (7)) is reorganized by regions such that

$$
\mathbf{D}(\omega)=\mathbf{K}-\omega^{2} \mathbf{M}=\left[\begin{array}{lll}
\mathbf{D}_{L L} & \mathbf{D}_{L R} & \mathbf{D}_{L I} \\
\mathbf{D}_{R L} & \mathbf{D}_{R R} & \mathbf{D}_{R I} \\
\mathbf{D}_{I L} & \mathbf{D}_{I R} & \mathbf{D}_{I I}
\end{array}\right]
$$

where $\mathbf{D}_{\alpha \beta}(\omega)=\mathbf{K}_{\alpha \beta}-\omega^{2} \mathbf{M}_{\alpha \beta}\left(\left\{()_{\alpha},()_{\beta}\right\} \in\left\{()_{L},()_{I},()_{R}\right\}\right)$. In absence of internal forces, Eq. (24) is recasted using static condensation as

$$
\left[\begin{array}{ll}
\mathfrak{D}_{L L} & \mathfrak{D}_{L R} \\
\mathfrak{D}_{R L} & \mathfrak{D}_{R R}
\end{array}\right]\left[\begin{array}{l}
\mathbf{u}_{L} \\
\mathbf{u}_{R}
\end{array}\right]=\left[\begin{array}{l}
\mathbf{f}_{L} \\
\mathbf{f}_{R}
\end{array}\right]
$$

where $\mathfrak{D}_{\alpha \beta}=\mathbf{D}_{\alpha \beta}-\mathbf{D}_{\alpha I} \mathbf{D}_{I I}^{-1} \mathbf{D}_{I \beta}$. Eq. (25) is rearranged to define a relation between opposite sides of the unit cell:

$$
\left[\begin{array}{r}
\mathbf{u}_{R} \\
-\mathbf{f}_{R}
\end{array}\right]=\left[\begin{array}{cc}
-\mathfrak{D}_{L R}^{-1} \mathfrak{D}_{L L} & \mathfrak{D}_{L R}^{-1} \\
\mathfrak{D}_{R R} \mathfrak{D}_{L R}^{-1} \mathfrak{D}_{L L}-\mathfrak{D}_{R L} & -\mathfrak{D}_{R R} \mathfrak{D}_{L R}^{-1}
\end{array}\right]\left[\begin{array}{l}
\mathbf{u}_{L} \\
\mathbf{f}_{L}
\end{array}\right]=\mathbf{T}\left[\begin{array}{l}
\mathbf{u}_{L} \\
\mathbf{f}_{L}
\end{array}\right]
$$

where $\mathbf{T}(\omega)$ is the transfer-matrix. Recasting Eq. (15) to

$$
\left[\begin{array}{l}
\mathbf{u}_{L} \\
\mathbf{f}_{L}
\end{array}\right]=e^{-\mu_{x}}\left[\begin{array}{cc}
\mathbf{N}_{\mathbf{X}}^{-1} & \mathbf{0} \\
\mathbf{0} & \mathbf{N}_{\mathbf{X}}^{-1}
\end{array}\right]\left[\begin{array}{c}
\mathbf{u}_{R} \\
-\mathbf{f}_{R}
\end{array}\right]
$$

its combination to Eq. (26) gives

$$
\left[\left[\begin{array}{cc}
\mathbf{N}_{\mathbf{X}}^{-1} & \mathbf{0} \\
\mathbf{0} & \mathbf{N}_{\mathbf{X}}^{-1}
\end{array}\right] \mathbf{T}(\omega)-\mathbf{I} e^{\mu_{x}}\right]\left[\begin{array}{l}
\mathbf{u}_{L} \\
\mathbf{f}_{L}
\end{array}\right]=\mathbf{0}
$$


Given a frequency $\omega$, associated propagation constants are found by solving the eigenvalue problem (28). However, for a large number of dofs, Eq. (28) might lead to spurious results due to ill-conditioning of the transfer-matrix [50]. Moreover, as it will be shown in Sec. 4.1.1, similar spurious results are observed when high-order-continuity at the boundary is enforced, even if the number of dof is small. To avoid this numerical issue, it is better to define the eigenvalue problem in terms of $\left(e^{\mu_{x}}+e^{-\mu_{x}}\right)$, and following the procedure detailed in $[44,50]$, one gets

$$
\left.\left[\begin{array}{cc}
\mathbf{N}_{\mathrm{X}}^{T} \mathfrak{D}_{R L} & \mathbf{0} \\
\mathbf{0} & \mathfrak{D}_{L R} \mathbf{N}_{\mathrm{X}}
\end{array}\right]-\frac{\operatorname{sech}\left(\mu_{x}\right)}{2}\left[\begin{array}{ll}
-\mathfrak{D}_{L L}-\mathbf{N}_{\mathrm{X}}^{T} \mathfrak{D}_{R R} \mathbf{N}_{\mathrm{X}} & +\mathbf{N}_{\mathrm{X}}^{T} \mathfrak{D}_{R L}-\mathfrak{D}_{L R} \mathbf{N}_{\mathrm{X}} \\
-\mathbf{N}_{\mathrm{X}}^{T} \mathfrak{D}_{R L}+\mathfrak{D}_{L R} \mathbf{N}_{\mathrm{X}} & -\mathfrak{D}_{L L}-\mathbf{N}_{\mathrm{X}}^{T} \mathfrak{D}_{R R} \mathbf{N}_{\mathrm{X}}
\end{array}\right]\right]\left[\begin{array}{l}
\mathbf{u}_{L} \\
\mathbf{u}_{L}
\end{array}\right]=\mathbf{0}
$$

where $\operatorname{sech}()$ is the hyperbolic secant function. The efficiency of this alternative approach together with the inverse method is demonstrated in a set of numerical problems in the next section, but before the case of patches meeting at kinks is investigated.

\subsection{Unit cells meeting at kinks}

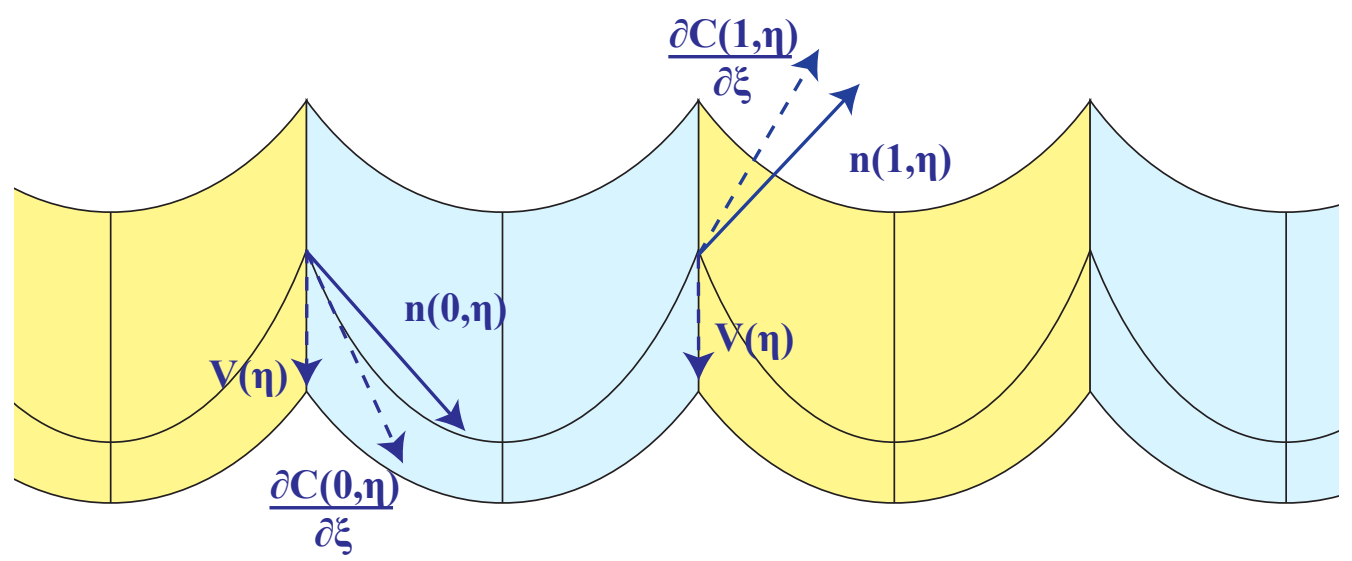

Figure 5: Translational periodic structure in the $\xi$ direction with unit cells meeting at kinks. Element boundaries in black lines. Note that for this specific configuration, the derivatives in the parametric space are not perpendicular to the edges.

When a kink is present at an interface between two patches, the angle formed by in-plane edge normals has to be preserved during the deformation (Fig. 5). 
Considering a kink parallel to the $\eta$ direction, the constraint of Eq. (11a) is replaced by

$$
\boldsymbol{n}(1, \eta)=e^{\mu_{x}} c_{0}(\eta) \mathbf{R}_{\theta, \boldsymbol{V}} \boldsymbol{n}(0, \eta),
$$

where $\boldsymbol{n}(\alpha, \eta)=\frac{\partial \boldsymbol{S}(\alpha, \eta)}{\partial \eta} \times\left(\frac{\partial \boldsymbol{S}(\alpha, \eta)}{\partial \xi} \times \frac{\partial \boldsymbol{S}(\alpha, \eta)}{\partial \eta}\right)\left(\alpha \in\left\{\begin{array}{ll}0,1 & 1\end{array}\right)\right.$ is normal to the patch interface and tangent to the surface. $\mathbf{R}_{\theta, \boldsymbol{V}}$ is the Rodrigues rotation matrix around the axis $\boldsymbol{V}=\frac{\partial \boldsymbol{S}(\alpha, \eta)}{\partial \eta} /\left\|\frac{\partial \boldsymbol{S}(\alpha, \eta)}{\partial \eta}\right\|$ by an angle of $\theta=$ atan2 $(\|\boldsymbol{n}(0, \eta) \times \boldsymbol{n}(1, \eta)\|, \boldsymbol{n}(0, \eta) \cdot \boldsymbol{n}(1, \eta))$ (Appendix A). Contrary to the case of the $\mathcal{G}^{1}$-continuity (Eq. (11a)), for the case of the kink, the angle of the rotation is fixed but not its axis, and its variation has to be accounted for in three-dimensional structures.

However, noting that $\boldsymbol{V}=\boldsymbol{n}(0, \eta) \times \boldsymbol{n}(1, \eta) /\|\boldsymbol{n}(0, \eta) \times \boldsymbol{n}(1, \eta)\|$ in presence of a kink, Eq. (30) is similar to Eq. (A.1) and it is shown in Appendix A that such problem is ill-posed. Indeed, this can be explained by the fact that the in-plane edge normals are not independent from the rotation axis.

To overcome this problem, the idea is to expressed $\boldsymbol{n}$ as a variation of the geometry with respect to the normal direction, such that Eq. (30) can be recasted to

$$
\frac{\partial \boldsymbol{S}(1, \eta)}{\partial n}=e^{\mu_{x}} c_{0}(\eta) \mathbf{R}_{\theta, \boldsymbol{V}} \frac{\partial \boldsymbol{S}(0, \eta)}{\partial n},
$$

where $c_{0}(\eta)=\left\|\frac{\partial \boldsymbol{S}(1, \eta)}{\partial n}\right\| /\left\|\frac{\partial \boldsymbol{S}(0, \eta)}{\partial n}\right\|$ and

$$
\frac{\partial \boldsymbol{S}(\alpha, \eta)}{\partial n}=\boldsymbol{n}(\alpha, \eta)=\sum_{i=1}^{n} \sum_{j=1}^{m}\left(\boldsymbol{\nabla} R_{i, j}^{p, k}(\alpha, \eta) \cdot \boldsymbol{n}(\alpha, \eta)\right) \boldsymbol{B}_{i, j}(\alpha, \eta)
$$

with $\boldsymbol{\nabla}=\left[\begin{array}{lll}\frac{\partial}{\partial x} & \frac{\partial}{\partial y} & \frac{\partial}{\partial z}\end{array}\right]^{T}$. After replacing $\boldsymbol{S}$, the initial geometry, by $\boldsymbol{S}+\boldsymbol{u}$, the deformed one, linearization of Eq. (31) assuming $\boldsymbol{u}$ small and keeping $\theta$ constant leads to

$$
\frac{\partial \boldsymbol{u}(1, \eta)}{\partial n}=e^{\mu_{x}} c_{0}(\eta)\left(\mathbf{R}_{\theta, \boldsymbol{V}} \frac{\partial \boldsymbol{u}(0, \eta)}{\partial n}-\delta \mathbf{R}_{\theta, \boldsymbol{V}} \frac{\partial \boldsymbol{S}(0, \eta)}{\partial n} \frac{\partial \boldsymbol{u}(0, \eta)}{\partial \eta}\right)
$$

where $\delta \mathbf{R}_{\theta, \boldsymbol{V}}$ is detailed in Eq. (A.3). Since $\frac{\partial \boldsymbol{u}(\alpha, \eta)}{\partial n}$ is derived with respect to the undeformed geometry, it is valid only for small deformations, an assumption which is anyway required for dispersion analysis with the Bloch theorem. 
While Eq. (33) has to be valid for all $\eta$, only $2 m$ equations can be imposed, where $m$ is the number of control points in the $\eta$ direction (Eq. (4)). To define the values of $\eta=\widehat{\eta}$ at which Eq. (33) is collocated, the Demko abscissae are used, following the approach of isogeometric collocation [51, 52]. They are obtained iteratively (command chbpnt in MATLAB) and are demonstrated to be stable. Otherwise, the Greville abscissae can be used but their stability is proved only for polynomial order smaller or equal than 3 [52].

Akin to Eqs. (13) and (14), Eqs. (10a) and (33) can be arranged in matrix form such that Eq. (18) is now a full matrix

$$
\left[\begin{array}{c}
\mathbf{u}_{R} \\
\mathbf{u}_{R B} \\
\mathbf{u}_{R T}
\end{array}\right]=e^{\mu_{x}}\left[\begin{array}{lll}
\mathbf{N}_{\mathrm{X} I I} & \mathbf{N}_{\mathrm{X} I B} & \mathbf{N}_{\mathrm{X} I T} \\
\mathbf{N}_{\mathrm{X} B I} & \mathbf{N}_{\mathrm{X} B B} & \mathbf{N}_{\mathrm{X} B T} \\
\mathbf{N}_{\mathrm{X} T I} & \mathbf{N}_{\mathrm{X} T B} & \mathbf{N}_{\mathbf{X} T T}
\end{array}\right]\left[\begin{array}{c}
\mathbf{u}_{L} \\
\mathbf{u}_{L B} \\
\mathbf{u}_{L T}
\end{array}\right] .
$$

Proceeding similarly in the $y$ direction, one gets:

$$
\left[\begin{array}{c}
\mathbf{u}_{T} \\
\mathbf{u}_{L T} \\
\mathbf{u}_{R T}
\end{array}\right]=e^{\mu_{y}}\left[\begin{array}{lll}
\mathbf{N}_{\mathrm{Y} I I} & \mathbf{N}_{\mathrm{Y} I L} & \mathbf{N}_{\mathrm{Y} I R} \\
\mathbf{N}_{\mathrm{Y} L I} & \mathbf{N}_{\mathrm{Y} L L} & \mathbf{N}_{\mathrm{Y} L R} \\
\mathbf{N}_{\mathrm{Y} R I} & \mathbf{N}_{\mathrm{Y} R L} & \mathbf{N}_{\mathrm{Y} R R}
\end{array}\right]\left[\begin{array}{c}
\mathbf{u}_{B} \\
\mathbf{u}_{L B} \\
\mathbf{u}_{R B}
\end{array}\right] .
$$

By solving the systems (34) and (35), Eq. (21) is updated with:

$$
\mathbf{A}(\boldsymbol{\mu})=\left[\begin{array}{cccc}
\mathbf{I} & \mathbf{0} & \mathbf{0} & \mathbf{0} \\
\mathbf{A}_{R \rightarrow L} & \mathbf{A}_{R \rightarrow B} & \mathbf{A}_{R \rightarrow L B} & \mathbf{0} \\
\mathbf{0} & \mathbf{I} & \mathbf{0} & \mathbf{0} \\
\mathbf{A}_{T \rightarrow L} & \mathbf{A}_{T \rightarrow B} & \mathbf{A}_{T \rightarrow L B} & \mathbf{0} \\
\mathbf{0} & \mathbf{0} & \mathbf{I} & \mathbf{0} \\
\mathbf{A}_{L T \rightarrow L} & \mathbf{A}_{L T \rightarrow B} & \mathbf{A}_{L T \rightarrow L B} & \mathbf{0} \\
\mathbf{A}_{R B \rightarrow L} & \mathbf{A}_{R B \rightarrow B} & \mathbf{A}_{R B \rightarrow L B} & \mathbf{0} \\
\mathbf{A}_{R T \rightarrow L} & \mathbf{A}_{R T \rightarrow B} & \mathbf{A}_{R T \rightarrow L B} & \mathbf{0} \\
\mathbf{0} & \mathbf{0} & \mathbf{0} & \mathbf{I}
\end{array}\right]
$$

where

$$
\begin{aligned}
& \mathbf{A}_{R \rightarrow L}=e^{\mu_{x}} \mathbf{N}_{\mathrm{X} I I}+e^{2 \mu_{x}+\mu_{y}} \mathbf{N}_{\mathrm{X} I T} \mathbf{I}_{\mathrm{YX}} \mathbf{N}_{\mathrm{Y} L R} \mathbf{N}_{\mathrm{X} B I}, \\
& \mathbf{A}_{R \rightarrow B}=e^{\mu_{x}+\mu_{y}} \mathbf{N}_{\mathrm{X} I T} \mathbf{I}_{\mathrm{YX}} \mathbf{N}_{\mathrm{Y} L I}, \\
& \mathbf{A}_{R \rightarrow L B}=e^{\mu_{x}} \mathbf{N}_{\mathrm{X} I B}+\mathbf{N}_{\mathrm{X} I T} \mathbf{I}_{\mathrm{YX}}\left[e^{2 \mu_{x}+\mu_{y}} \mathbf{N}_{\mathrm{Y} L R} \mathbf{N}_{\mathrm{X} B B}+e^{\mu_{x}+\mu_{y}} \mathbf{N}_{\mathrm{Y} L L}\right], \\
& \mathbf{A}_{T \rightarrow L}=e^{\mu_{x}+\mu_{y}} \mathbf{N}_{\mathrm{Y} I R} \mathbf{I}_{\mathrm{XY}} \mathbf{N}_{\mathrm{X} B I}, \\
& \mathbf{A}_{T \rightarrow B}=e^{\mu_{y}} \mathbf{N}_{\mathrm{Y} I I}+e^{\mu_{x}+2 \mu_{y}} \mathbf{N}_{\mathrm{Y} I R} \mathbf{I}_{\mathrm{XY}} \mathbf{N}_{\mathrm{X} B T} \mathbf{N}_{\mathrm{Y} L I}, \\
& \mathbf{A}_{T \rightarrow L B}=e^{\mu_{y}} \mathbf{N}_{\mathrm{Y} I L}+\mathbf{N}_{\mathrm{Y} I R} \mathbf{I}_{\mathrm{XY}}\left[e^{\mu_{x}+2 \mu_{y}} \mathbf{N}_{\mathrm{X} B T} \mathbf{N}_{\mathrm{Y} L L}+e^{\mu_{x}+\mu_{y}} \mathbf{N}_{\mathrm{X} B B}\right],
\end{aligned}
$$


$\mathbf{A}_{L T \rightarrow L}=e^{\mu_{x}+\mu_{y}} \mathbf{I}_{\mathrm{YX}} \mathbf{N}_{\mathrm{Y} L R} \mathbf{N}_{\mathrm{X} B I}$,

$\mathbf{A}_{L T \rightarrow B}=e^{\mu_{x}} \mathbf{I}_{\mathrm{YX}} \mathbf{N}_{\mathrm{Y} L I}$,

$\mathbf{A}_{L T \rightarrow L B}=\mathbf{I}_{\mathrm{YX}}\left[e^{\mu_{x}+\mu_{y}} \mathbf{N}_{\mathrm{Y} L R} \mathbf{N}_{\mathrm{X} B B}+e^{\mu_{x}} \mathbf{N}_{\mathrm{Y} L L}\right]$,

$\mathbf{A}_{R B \rightarrow L}=e^{\mu_{x}} \mathbf{I}_{\mathrm{XY}} \mathbf{N}_{\mathrm{X} B I}$,

$\mathbf{A}_{R B \rightarrow B}=e^{\mu_{x}+\mu_{y}} \mathbf{I}_{\mathrm{XY}} \mathbf{N}_{\mathrm{X} B T} \mathbf{N}_{\mathrm{Y} L I}$,

$\mathbf{A}_{R B \rightarrow L B}=\mathbf{I}_{\mathrm{XY}}\left[e^{\mu_{x}+\mu_{y}} \mathbf{N}_{\mathrm{X} B T} \mathbf{N}_{\mathrm{Y} L L}+e^{\mu_{x}} \mathbf{N}_{\mathrm{X} B B}\right]$,

$\mathbf{A}_{R T \rightarrow L}=e^{\mu_{x}} \mathbf{N}_{\mathrm{X} T I}+e^{2 \mu_{x}+\mu_{y}} \mathbf{N}_{\mathrm{X} T T} \mathbf{I}_{\mathrm{YX}} \mathbf{N}_{\mathrm{Y} L R} \mathbf{N}_{\mathrm{X} B I}$,

$\mathbf{A}_{R T \rightarrow B}=e^{\mu_{x}+\mu_{y}} \mathbf{N}_{\mathrm{X} T T} \mathbf{I}_{\mathrm{YX}} \mathbf{N}_{\mathrm{Y} L I}$,

$\mathbf{A}_{R T \rightarrow L B}=e^{\mu_{x}} \mathbf{N}_{\mathrm{X} T B}+\mathbf{N}_{\mathrm{X} T T} \mathbf{I}_{\mathrm{YX}}\left[e^{2 \mu_{x}+\mu_{y}} \mathbf{N}_{\mathrm{Y} L R} \mathbf{N}_{\mathrm{X} B B}+e^{\mu_{x}+\mu_{y}} \mathbf{N}_{\mathrm{Y} L L}\right]$,

$\mathbf{A}_{R T \rightarrow L}=e^{\mu_{x}+\mu_{y}} \mathbf{N}_{\mathrm{Y} R R} \mathbf{I}_{\mathbf{X Y}} \mathbf{N}_{\mathrm{X} B I}$,

$\mathbf{A}_{R T \rightarrow B}=e^{\mu_{y}} \mathbf{N}_{\mathrm{Y} R I}+e^{\mu_{x}+2 \mu_{y}} \mathbf{N}_{\mathrm{Y} R R} \mathbf{I}_{\mathrm{XY}} \mathbf{N}_{\mathrm{X} B T} \mathbf{N}_{\mathrm{Y} L I}$,

$\mathbf{A}_{R T \rightarrow L B}=e^{\mu_{y}} \mathbf{N}_{\mathrm{Y} R L}+\mathbf{N}_{\mathrm{Y} R R} \mathbf{I}_{\mathrm{XY}}\left[e^{\mu_{x}+2 \mu_{y}} \mathbf{N}_{\mathrm{X} B T} \mathbf{N}_{\mathrm{Y} L L}+e^{\mu_{x}+\mu_{y}} \mathbf{N}_{\mathrm{X} B B}\right]$,

and

$$
\begin{aligned}
& \mathbf{I}_{\mathrm{YX}}=\left[\mathbf{I}-e^{\mu_{x}+\mu_{y}} \mathbf{N}_{\mathrm{Y} L R} \mathbf{N}_{\mathrm{X} B T}\right]^{-1}, \\
& \mathbf{I}_{\mathrm{XY}}=\left[\mathbf{I}-e^{\mu_{x}+\mu_{y}} \mathbf{N}_{\mathrm{X} B T} \mathbf{N}_{\mathrm{Y} L R}\right]^{-1} .
\end{aligned}
$$

Note that similarly to the system (18), the system (34) is composed of 5 unknowns and 6 equations. However, the 6 equation are now not linearly independent, and two different expressions for the term $\mathbf{u}_{R T}$ are found (Eqs. (37e) and (37f)). In spite of this, the use of Eq. (37e) and (37f) provides almost identical results which made not possible to establish which one to choose. The average of both terms appeared to be the most reasonable choice and has been employed in this work.

\section{Numerical Results}

In this section, structural slender beam and shell problems are presented, and the following formulations are used. Beams are modeled by isogeometric planar extensible-elastica elements [9], whereas shells are modeled using isogeometric Kirchhoff-Love elements [14]. These both theories account for axial/membrane and bending strains, but neglect shear ones, in agreement 
with the assumption of slenderness. They have also the advantage of being rotation-free such that only three and two dofs per control point are required in the case of shells and planar beams, respectively. While the Galerkin method is used, bending strains in the weak form are described by second-order derivatives of the displacement field, such that the NURBS basis functions have to be at least $\mathcal{C}^{1}$-continuous inside the domain.

If not specified, the following material notation and numerical values are used: the Young's modulus is $E=70 \mathrm{GPa}$, and the density $\rho=2700 \mathrm{~kg} \mathrm{~m}^{-3}$. In addition, for shells, $\nu=0.33$ is the Poisson ratio and $G=E /(2(1-\nu))$ is the shear modulus.

In order to compare numerical results with the theoretical convergence order, the following approach is used. For a given eigenvalue problem where $\lambda_{k}^{h}$ is the $k^{\text {th }}$ approximated eigenvalue ( $h$ being the mesh size), the error can be estimated by $[53-56]$

$$
\frac{\left|\lambda_{k}-\lambda_{k}^{h}\right|}{\left|\lambda_{k}\right|} \leq C\left(h \lambda_{k}^{1 /(2 m)}\right)^{\beta},
$$

where $\lambda_{k}$ is the exact solution, $C$ a constant, and $\beta=2(p+1-m)$ the estimated convergence order, with $p$ the basis order and $m$ the problem order in the weak form ( $2 m$ being the PDE order).

\subsection{Quasi-one-dimensional wave propagation}

\subsubsection{Ring}

In this first example, a slender planar ring is investigated and modeled using isogeometric extensible-elastica, which have the advantage to represent exactly the curvature. Dispersion is analyzed using the revisited Bloch theorem, and instead of modeling $2 \pi$ of the circumference, corresponding to the periodicity by translation, rotational symmetries are accounted for, such that only a small angle $\theta$ of the ring is modeled (Fig. 6). This reduction of the problem is possible after updating the boundary conditions [57], and consists in replacing the identity matrix $\mathbf{I}_{d}$ in Eq. (16) by the transpose of the rotation matrix

$$
\mathbf{R}_{\mathbf{o t} \theta}^{T}=\left[\begin{array}{cc}
\cos \theta & -\sin \theta \\
\sin \theta & \cos \theta
\end{array}\right]
$$




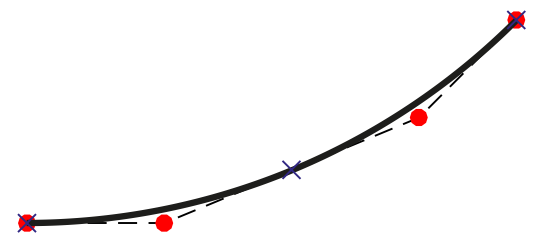

Figure 6: Portion $\theta=\pi / 4$ of a circle described by a NURBS curve of polynomial order $p=2$ and $h$-refined with 2 elements.

Numerical dispersion curves are shown for $r=10 t$ and $\theta=2 \pi / 32$ in Fig. 7, and compared to an analytical dispersion equation derived from beam theory [8]:

$$
\kappa^{6}-\left(\kappa_{0}^{2}+\frac{2}{r^{2}}\right) \kappa^{4}-\left(\beta^{4}+\frac{\kappa_{0}^{2}}{r^{2}}-\frac{1}{r^{4}}\right) \kappa^{2}+\left(\kappa_{0}^{2}-\frac{1}{r^{2}}\right) \beta^{4}=0,
$$

where $\kappa=\theta r \mu_{x}$ is the wavenumber, $\kappa_{0}^{2}=\omega^{2} \rho / E, \beta^{4}=\omega^{2} \rho A /(E I), r$ the ring radius, and $t=1 \mathrm{~mm}$ the thickness. For a square cross section, the present case, $A=t^{2}$ is the area, and $I=t^{4} / 12$ the area-moment of inertia.
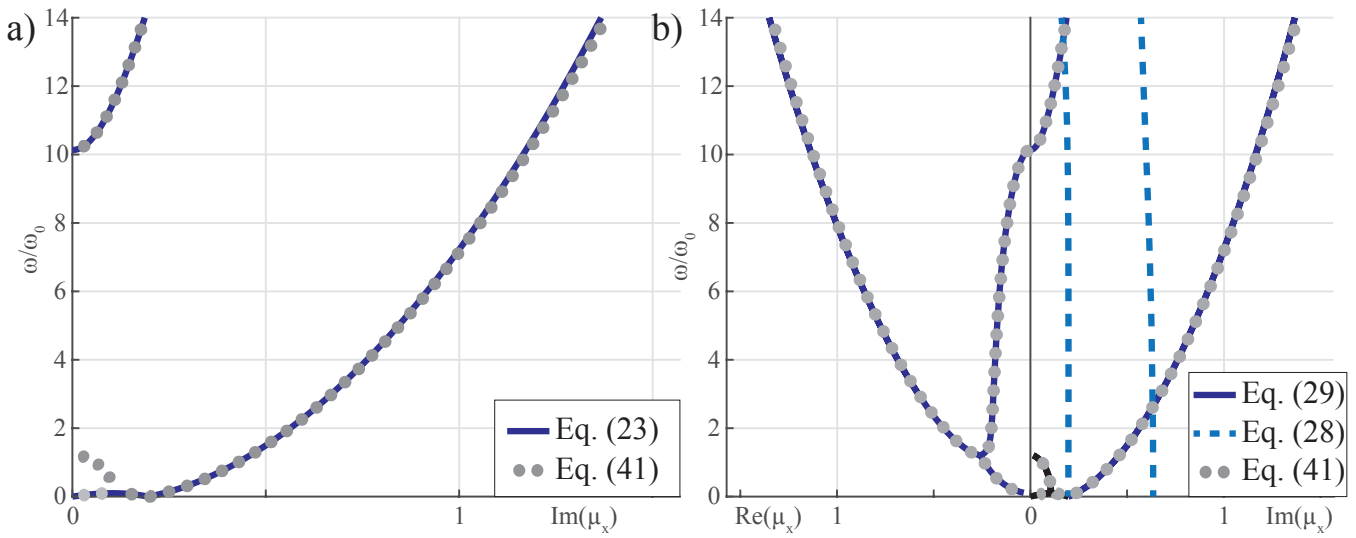

Figure 7: Dispersion relation for the ring normalized by $\omega_{0}=\pi^{2} \sqrt{E / \rho}$, obtained with the inverse (Eq. (23)) (a) and direct (Eqs. (29) and (28)) (b) methods, and compared to the analytical Eq. (41). Quadratic NURBS basis is used and discretized by 2 elements (Fig. 6).

For the dispersion curves obtained with the inverse method (Eq. (23)), results match analytical solutions, except for one branch which is evanescent $\left(\operatorname{Re}\left(\mu_{x}\right) \neq 0\right)$, and therefore, cannot be captured by this method (Fig. 7a). 


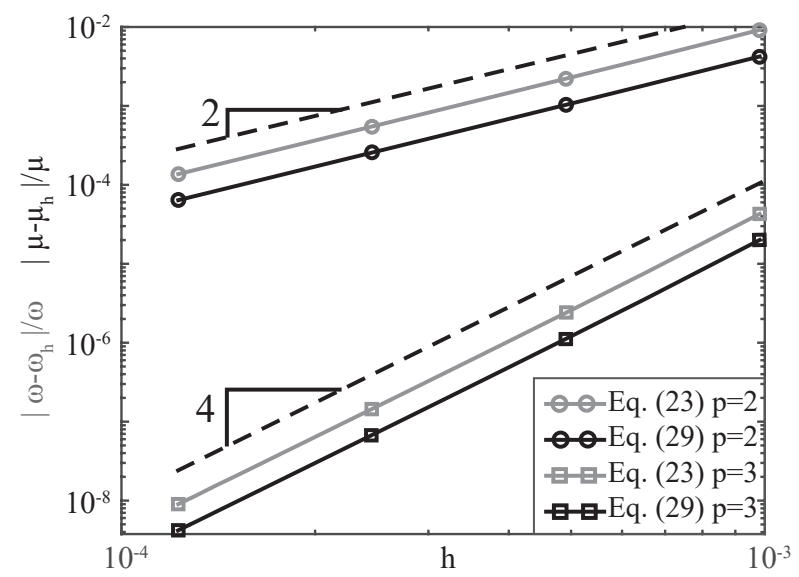

Figure 8: Error versus mesh size $h$ for the inverse (Eq. (23)) and direct (Eq. (29)) methods, at $\mu_{x}=\mathrm{i}$ and $\omega\left(\mu_{x}=\mathrm{i}\right)$, in gray and black lines, respectively, and with $p=2$ and $p=3$ with round and square markers, respectively. Convergence order estimates (Eq. (39)) for $m=2$ and $p=\{2,3\}$ in dashed lines.

Indeed, Eq. (23) is derived assuming a purely propagating wave $\left(\operatorname{Re}\left(\mu_{x}\right)=0\right)$. For the transfer-matrix approach, if Eq. (28) is used (dashed lines in Fig. 7b), spurious results are obtained. While the number of dof is small (only 8 dofs), the ill-conditioning of the eigenvalue problem results from the $\mathcal{C}^{1}$-continuity constraint. Indeed, the same simulation performed with classical FE and for the same number of dof gives accurate results, as shown in [57]. To avoid ill-conditioning, Eq. (29) is used instead and analytical results are this time recovered (full lines in Fig. 7b).

Convergence plots are shown in Fig. 8, for the inverse and well-posed direct Bloch method with different NURBS basis orders. Convergence orders are found in agreement with convergence estimates (Eq. (39)), validating the proposed method for this example.

\subsubsection{Longitudinally-wrinkled helicoid}

Twisted and stretched elastic ribbons handle multiple morphological instabilities $[58,59]$ and we focus here on the longitudinally-wrinkled helicoids (Fig. 9a). For some specific configurations, while the global structure is in tension, twisting the ribbon compresses regions close to the centroid both in the longitudinal and radial directions, such that a periodic buckled pattern can be obtained (ripples). In the present paper, a steel ribbon from Brütsch \& Rüegger is used and has the following characteristics: width $W=12.7$ 
mm, thickness $t=0.04 \mathrm{~mm}$, length $L=25 \mathrm{~cm}$, Young Modulus $E=210$ GPa, Poisson ratio $\nu=0.3$, and density $\rho=7800 \mathrm{~kg} \mathrm{~m}^{-3}$. In order to get the first longitudinally-wrinkled mode, the strip is stretched by a tension $T=67 \mathrm{~N}$ then twisted by an angle $\Theta=\pi$ (Fig. 9b). The thin ribbon is modeled by isogeometric Kirchhoff-Love elements [14] capable of handling large geometrically-nonlinear deformations. Quadratic NURBS basis functions are used and the domain is discretized by 30 and 300 elements in the radial and longitudinal directions, respectively. Simulation results are shown in Fig. 9c,d and we refer the reader to [60] for more details.
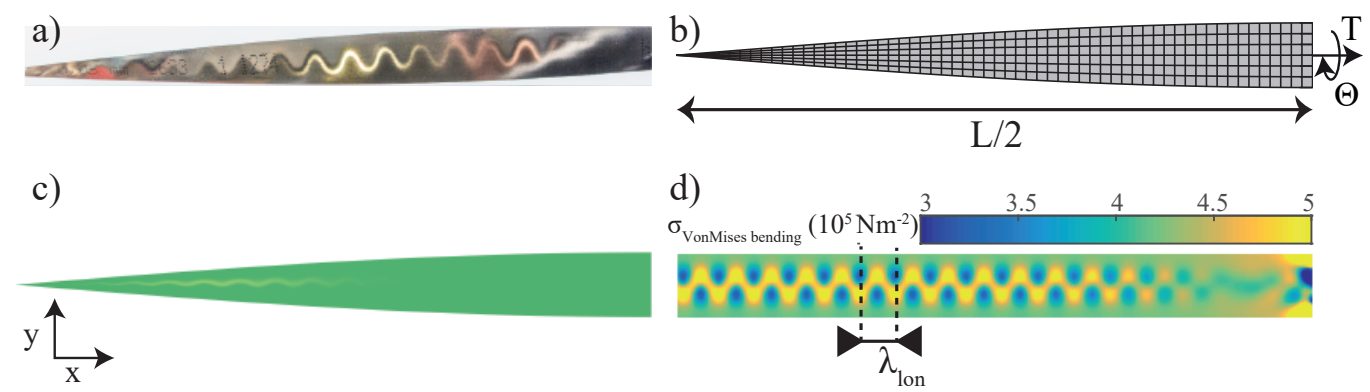

Figure 9: Experimental test (a), coarse numerical model (b), numerical deformed model (c), and numerical Von Mises bending stress projected in the undeformed configuration (d) of the longitudinally-wrinkled helicoid. Only half of the specimens are represented.

Similarly to the tetrahelix studied in [57], the longitudinally-wrinkled helicoid in general does not possess purely translational symmetries (the helix step over the buckling period is in general not a rational number). However, a screw symmetry is present (a translation combined to a rotation) and to account for it, $\mathbf{I}_{d}$ in Eq. (16) is replaced by the transpose of the rotation matrix $[57,61]$

$$
\mathbf{R}_{\mathbf{o t} \theta}^{T}=\left[\begin{array}{ccc}
1 & 0 & 0 \\
0 & \cos \theta & -\sin \theta \\
0 & \sin \theta & \cos \theta
\end{array}\right]
$$

where $\theta=\Theta \lambda_{\text {lon }} / L$ and $\lambda_{\text {lon }}$ the buckling period (Fig. 9). Note that the period of the screw symmetry can be still reduced by a factor 2 and we refer the reader to [61] for more details.

Dispersion curves are shown in Fig. 10, and the main finding is that the periodic deformation couples longitudinal and flexural modes, such that 
longitudinal waves become dispersive. As a side note, it is shown in [60] that dispersion of longitudinal waves is a key component in the formation of solitary waves and its quantification with the Bloch theorem is fundamental for analytical wave characterizations.

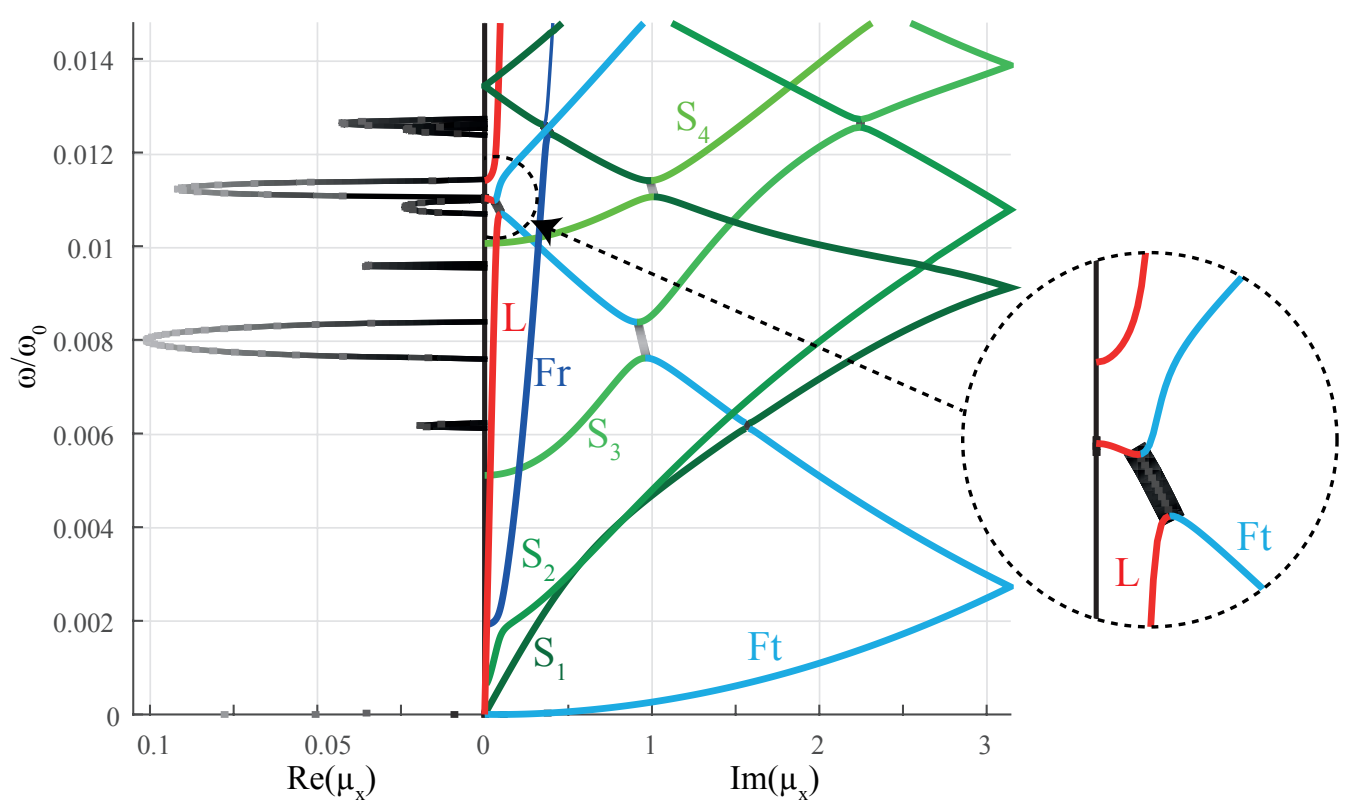

Figure 10: (a) Dispersion curves for the longitudinally-wrinkled helicoid obtained with Eq. (29) and normalized by $\omega_{0}=\frac{2 \pi}{l} \sqrt{E / \rho}$. Purely propagating branches corresponding to longitudinal $(\mathrm{L})$, transverse/radial flexural $(\mathrm{Ft} / \mathrm{Fr})$, and shear $\left(\mathrm{S}_{i}\right)$ modes $(i$ denotes the mode number) in color lines and evanescent modes in gray shades, from black to white lines corresponding to $\operatorname{Re}\left(\mu_{x}\right)=\left[\begin{array}{ll}0 & 0.15\end{array}\right]$.

\subsection{Quasi-two-dimensional wave propagation}

For quasi-two-dimensional wave propagation, dispersion curves are shown in the contour of the irreducible Brillouin zone (IBZ) defined by the triangle

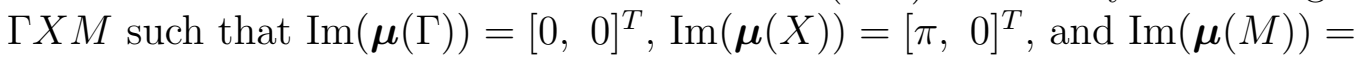
$[\pi, \pi]^{T}[4]$ (Fig. 11).

\subsubsection{Thin flat shell}

An infinite thin flat shell is investigated in this example using four different unit cells, allowing investigations of a nonuniform knot vector, a curved contour, and derivatives in the parametric space which are not aligned (Fig. 12). 


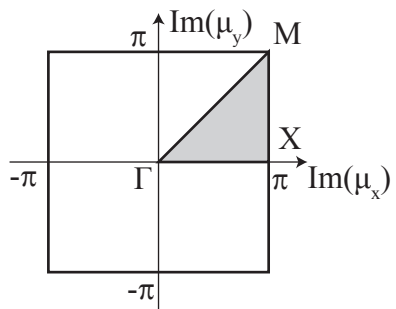

Figure 11: First Brillouin zone and irreducible Brillouin zone in gray.

These flat surfaces are generated by taking the tensor product of two identical planar B-spline curves rotated each other by the angle $\pi / 2$. Parameters of the B-spline curves are given in Table 1 for each configuration.

Table 1: B-spline curve parameters from which B-spline surfaces of Fig. 12 are generated. $\Xi$ is the knot vector and $\boldsymbol{B}_{x}$ and $\boldsymbol{B}_{y}$ are respectively the $x$ and $y$ components of the control point coordinates. $\xi_{\log 1}=1-\log (2(\exp -1) / 3+1)$ and $\xi_{\log 2}=1-\log ((\exp -1) / 3+1)$. B-spline basis order is $p=2$.

\begin{tabular}{|c|c|c|c|}
\hline Shape & $\Xi$ & $\overline{B_{x}}$ & $\overline{B_{y}}$ \\
\hline Fig. $12 \mathrm{a}$ & $\left\{\begin{array}{lllllllll}0 & 0 & 0 & 1 / 3 & 2 / 3 & 1 & 1 & 1\end{array}\right\}$ & $\{0 l / 6 l / 25 l / 6 l\}$ & $\left\{\begin{array}{lllll}0 & 0 & 0 & 0 & 0\end{array}\right\}$ \\
\hline Fig. $12 \mathrm{~b}$ & 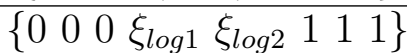 & $\{0 l / 6 l / 25 l / 6 l\}$ & $\left\{\begin{array}{lllll}0 & 0 & 0 & 0 & 0\end{array}\right\}$ \\
\hline Fig. 12c & 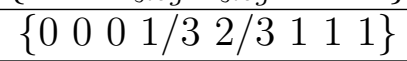 & $\{0 l / 6 l / 25 l / 6 l\}$ & $\{0-l / 80 l / 80\}$ \\
\hline Fig. $12 \mathrm{~d}$ & 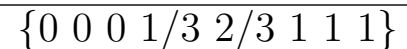 & $\{0 l / 6 l / 25 l / 6 l\}$ & $\left\{\begin{array}{lllll}0 & 0 & 0 & l / 8 & 0\end{array}\right\}$ \\
\hline
\end{tabular}

The dispersion curves obtained from the revisited Bloch method are given in Fig. 13a for a period $l=0.1 \mathrm{~m}$. Longitudinal ('L'), in-plane shear ('S'), and flexural ('F') branches are found in agreement with analytical dispersion equations [8]

$$
\begin{aligned}
\omega_{L} & =\sqrt{\frac{E}{\left(1-\nu^{2}\right) \rho}} \kappa, \\
\omega_{F} & =\sqrt{\frac{E t^{2}}{12\left(1-\nu^{2}\right) \rho}} \kappa^{2}, \\
\omega_{S} & =\sqrt{\frac{G}{\rho} \kappa .}
\end{aligned}
$$

Note that in Fig. 12a-c, the control points at the boundary are collinear such that the simplified Eq. (21) can be used, which is not the case of Fig. 12d 
a)

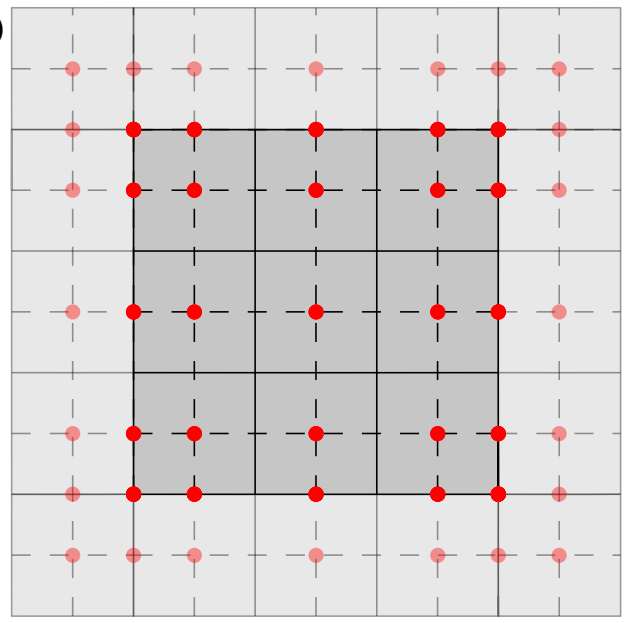

c)

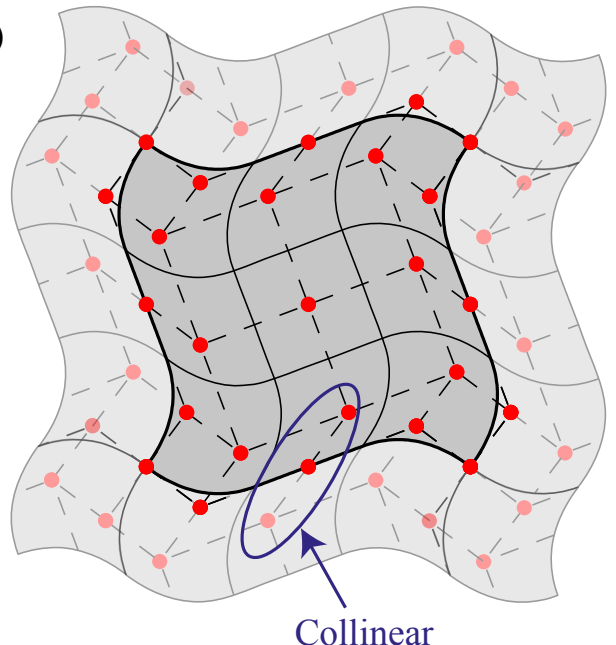

b)

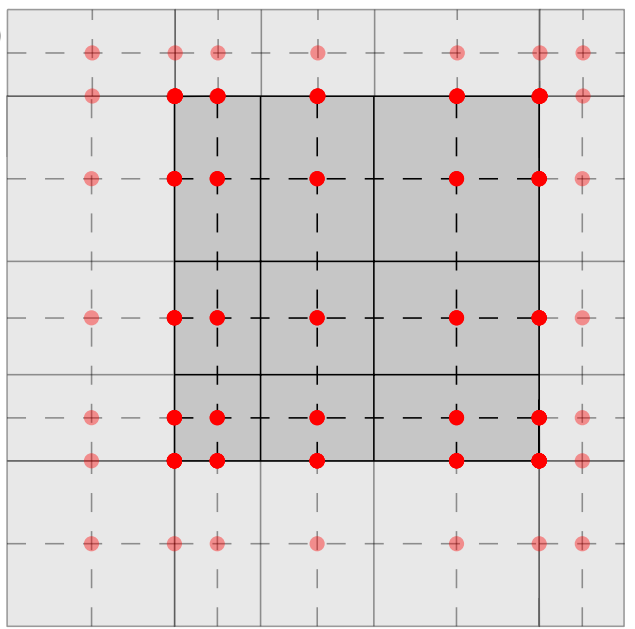

d)

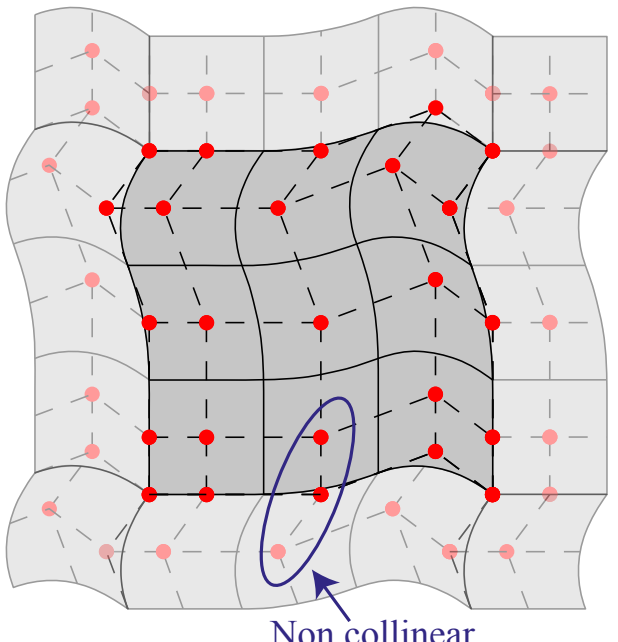

Figure 12: (a-d) Flat periodic unit cell in dark gray surrounded by one element width of the rest of the periodic pattern. B-spline surfaces are constructed from the tensor product of two perpendicular B-spline curves. These curves are different in (a-d) and their parametrization is given in Table 1.

where the full Eq. (36) is required. Convergence orders for the four configurations are then compared to the error estimate in Fig. 14. It is found that for longitudinal/shear and flexural modes, the convergence orders are $\beta=4$ and $\beta=2$, respectively. This is in agreement with the fact that the axial/shear strains are first-order problems into the weak form $(m=1)$, whereas bending deformations involve second-order derivatives $(m=2)$ (Eq. (39)). 

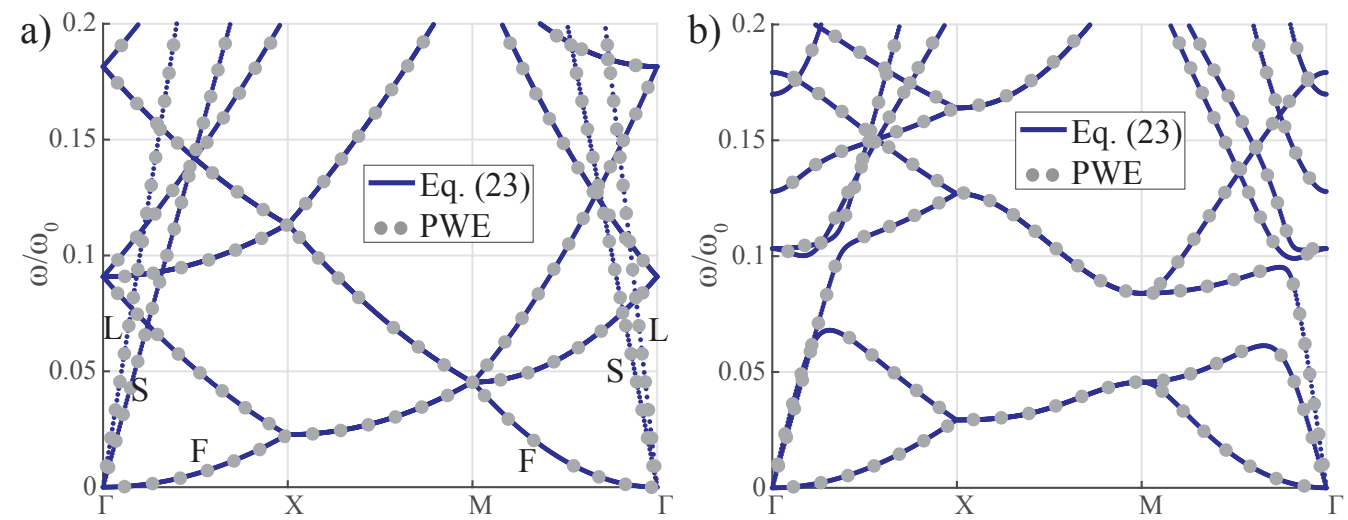

Figure 13: Dispersion curves normalized by $\omega_{0}=\frac{2 \pi}{l} \sqrt{\frac{E}{\left(1-\nu^{2}\right) \rho}}$ for flat (Fig. 12a) (a) and undulated (Fig. 15) (b) shells in the IBZ contour. Full and dotted lines are obtained with Eq. (23) and the PWE method [62], respectively. These plots are shown for a refinement of 32 subdivisions in both directions.

a)

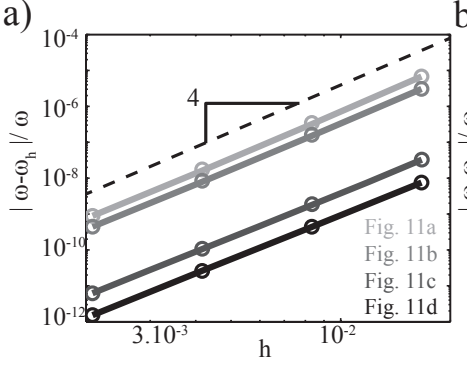

b)

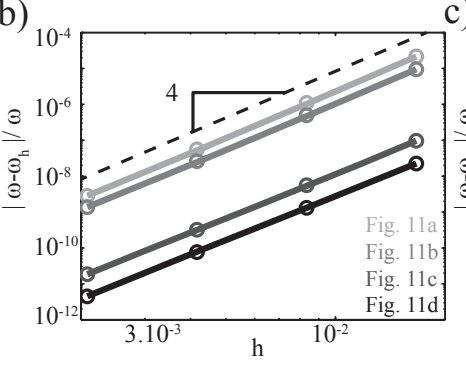

c)

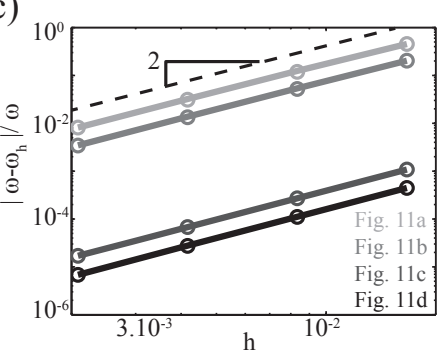

Figure 14: Dispersion frequency error versus mesh size $h$ of a flat shell for axial (a), shear (b) and bending (c) branches at $\mu_{x}=\mu_{y}=3 \mathrm{i} \pi / 4$. Considered unit cells are shown in Fig. 12a-d and are represented here from black to light gray lines, respectively. Convergence order estimates (Eq. (39)) with $m=1(\mathrm{a}, \mathrm{b})$ and $m=2(\mathrm{c})$ in dashed lines.

\subsubsection{Undulated shell}

In this example, an undulated shell is investigated where a point $\boldsymbol{x}_{s}=$ $\left\{x_{s}, y_{s}, z_{s}\right\}$ belonging to the surface is defined by

$$
z_{s}\left(x_{s}, y_{s}\right)=\frac{z_{0}}{2}\left(\cos \left(\frac{2 \pi}{l} x_{s}\right)+\cos \left(\frac{2 \pi}{l} y_{s}\right)\right),
$$

where $l$ and $z_{0}$ are the undulation period and magnitude, respectively. Since the surface is expressed through a transcendental function [63], it cannot be exactly described by a NURBS surface. Hence an approximation is found by 
projecting the flat surface described in Fig. 12a on the undulated geometry and minimizing the error in a least-square sense [47].

The resulting geometry is shown in Fig. 15 for $l=0.1 \mathrm{~m}, \zeta=z_{0} / l=$ 0.05 and $\gamma=t / l=0.05$, and corresponding dispersion curves are shown in Fig. 13b and are found in good agreement with plane wave expansion (PWE) method [62]. However, this method assumes orthogonality between the lines of the principal curvature, and therefore, it cannot be used as an exact reference for convergence purpose.

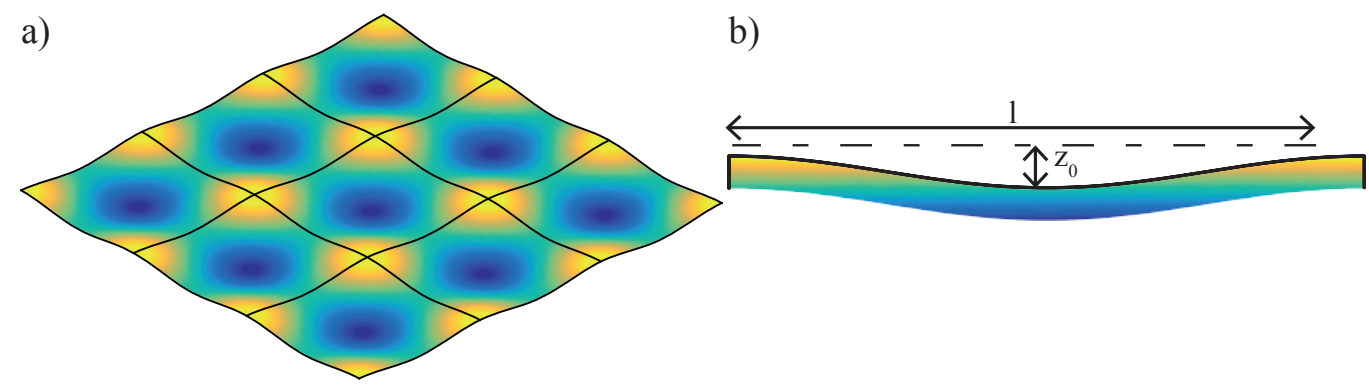

Figure 15: Undulated shell composed of 9 unit cells (a), and with detailed view of one profile (b).

As a side note, it is shown in [61] that the minimum period by translation is the one considered here, but that the unit cell used in the Bloch theorem can be reduced by two considering the two glide symmetries of this structure.

\subsubsection{Repeated dome}

In this example, we consider a three-dimensional structure with unit cells meeting at kinks: the repeated dome shown in Fig. 16a. Similarly to the undulated shell, the geometry is modeled with isogeometric Kirchhoff-Love shells, and is generated using the cosine function, but taking its absolute value such that

$$
z_{s}\left(x_{s}, y_{s}\right)=\frac{z_{0}}{2}\left|\cos \left(\frac{\pi}{l} x_{s}\right) \cos \left(\frac{\pi}{l} y_{s}\right)\right|
$$

where in the present case, $l=0.1 \mathrm{~m}$ and $z_{0}=0.25 l$. The shell thickness is $t=0.02 l$ and the geometry is discretized by quadratic B-splines with 32 elements in both directions. Since units cells meet at kinks, Eq. (36) is used to obtain dispersion curves (Fig. 16c), and results are compared to a solution computed with the commercial software $N X$ Nastran. Results are found in 
perfect agreement, except at high frequency, and is explained by the fact that Reissner-Mindlin plate elements are used in $N X$ Nastran, which account for shear deformations, contrary to the Kirchhoff-Love theory.

To show that the variation of the rotation matrix should be accounted for, the dispersion curves are also given when $\delta \mathbf{R}_{\theta, \boldsymbol{V}}$ is neglected in Eq. (33), and as expected, results do not converge to the correct solution.
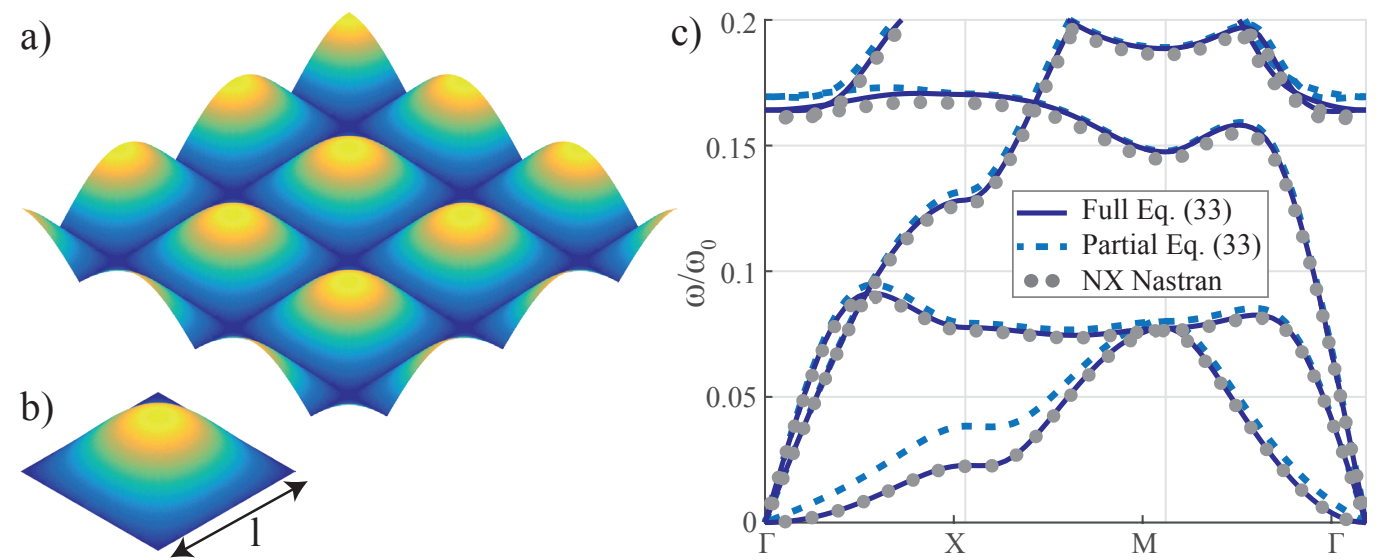

Figure 16: Repeated dome (a) with unit cells (b) meeting at kinks and resulting dispersion curves normalized by $\omega_{0}=\frac{2 \pi}{l} \sqrt{\frac{E}{\left(1-\nu^{2}\right) \rho}}$ in the IBZ contour (c). Full and dashed lines are obtained using Eq. (33), with and without considering variation of the rotation matrix, respectively. Dotted lines are obtained with $N X$ Nastran.

\subsubsection{Expanded metal sheet}

As a last example, we demonstrate the possibility to use multi-patch NURBS surfaces inside a periodic unit cell by investigating expanded metal sheets (Fig. 17). This structure is widely used in engineering [64], but to the best of the author's knowledge, it has not been investigated from a wave propagation point of view. Note however the dispersion analysis of kirigami pyramidal cores in [49], a structure constructed from expanded metal sheets.

One period of the specimen geometry is approximately reproduced from the aluminum stock number E312081S from Metals Depot ${ }^{\circledR}$, such that the diamond profile shown in Fig. 17d is $l=24 \mathrm{~mm}$ long, $h=10 \mathrm{~mm}$ high, $b=8$ $\mathrm{mm}$ deep, and the plate from which the structure is manufactured is $t=2$ $\mathrm{mm}$ thick. The half length of the common interface should be $r=l / 5$ but is in reality reduced by $e=r / 3$ due to the presence of a chamfer. 

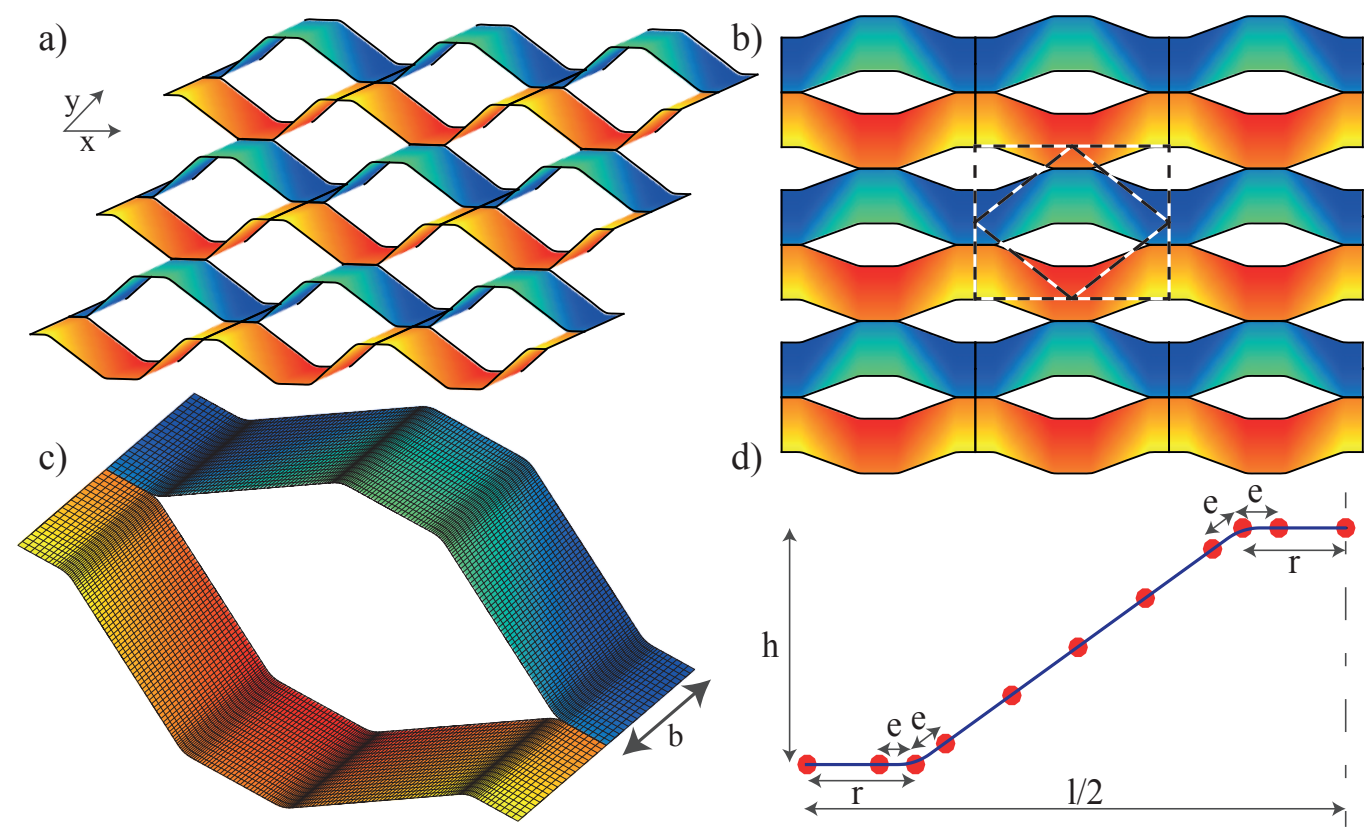

Figure 17: Expanded metal sheet (a) viewed from top (b) with the periodic unit cell composed of two patches (c), for which the profile is modeled by a B-spline curve (d).

To model the cut inside the unit cell, two bivariate B-spline patches are used such that the diamond is divided into lower and upper parts. KirchhoffLove shell elements are used and $\mathcal{G}^{1}$-continuity between the two patches is maintained enforcing the collinearity of adjacent control points [14]. The $\mathrm{B}$-spline basis order is $p=2$ and each knot vector is $h$-refined by 10 and 20 elements in the longitudinal and depth directions, respectively.

Noting that adjacent control points are collinear, Eq. (21) is used and resulting dispersion curves are shown in Fig. 18. Note finally that the translation period used in the present work (dashed rectangle in Fig. 18b) could have been replaced by a smaller one (dashed diamond in Fig. 18b) to reduce the size of the eigenvalue problem [61]. However, this choice is justified by the difficulties in modeling the smallest period with NURBS patches.

\section{Conclusions}

Wave propagation in periodic problems governed by high-order PDEs and discretized by NURBS-based IGA is investigated by revisiting the boundary condition of the Bloch theorem. Whereas the $\mathcal{G}^{1}$-continuity is enforced by 


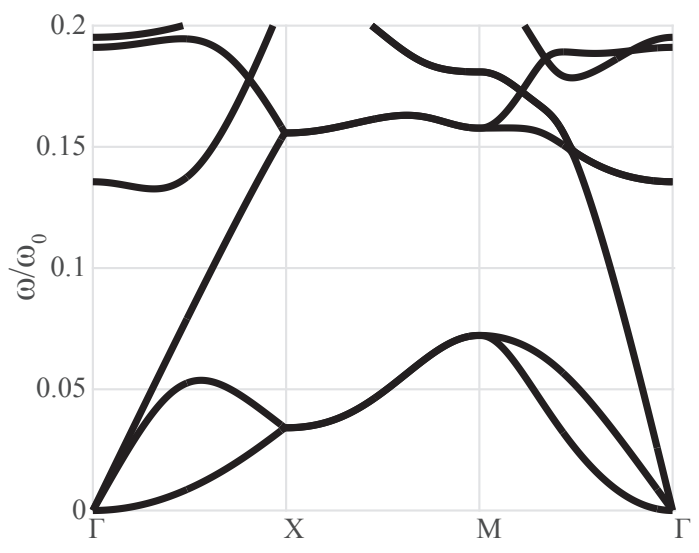

Figure 18: Dispersion curves normalized by $\omega_{0}=\frac{2 \pi}{l} \sqrt{\frac{E}{\left(1-\nu^{2}\right) \rho}}$ for the expanded metal sheet shown in Fig. 17 in the IBZ contour.

applying periodic boundary conditions to maintain the collinearity between adjacent control points, a different procedure is used in case of unit cells meeting at kinks. The angle of the kink is maintained by introducing a rotation matrix between variations of the field with respect to the in-plane edge normals. These conditions are collocated at the Demko abscissae, commonly used in the isogeometric collocation method. While the direct method of the Bloch theorem is derived for quasi-two-dimensional wave propagation, only the propagating wave is captured with this method. Evanescent waves in quasi-one-dimensional structures are obtained with the transfer-matrix approach. The applicability of the proposed method is shown in a set of different numerical problems, and it is found that the convergence order of the dispersion is in agreement with error estimates, validating the present work.

\section{Acknowledgments}

The Research Fund KU Leuven is gratefully acknowledged for its support. This research was also partially supported by Flanders Make, the strategic research centre for the manufacturing industry. The research of F. Maurin is funded by an Experienced Researcher grant within the European ANTARES Project, under the FP7 Marie Curie Programme (GA606817). The research of L. Coox is funded by a grant from the IWT Flanders. The IWT Flanders within the OPTIWIND project is gratefully acknowledged for its support. 
The research of E. Deckers is funded by a grant from the Fund for Scientific Research - Flanders (F.W.O.).

\section{Appendix A. The Rodrigues rotation formula}

Given $\boldsymbol{W}_{1}$ and $\boldsymbol{W}_{2}$ two arbitrary unit vectors, a rotation matrix $\mathbf{R}_{\theta, \boldsymbol{V}}$ exists such that

$$
\boldsymbol{W}_{2}=\mathbf{R}_{\theta, \boldsymbol{V}} \boldsymbol{W}_{1}
$$

where [38]

$$
\mathbf{R}_{\theta, \boldsymbol{V}}=\cos \theta \mathbf{I}+\sin \theta[\boldsymbol{V}]_{\times}+(1-\cos \theta) \boldsymbol{V} \boldsymbol{V}^{T}
$$

and with $[\boldsymbol{a}]_{\times} \boldsymbol{b}=\boldsymbol{a} \times \boldsymbol{b}$ the cross product expressed as a matrix product

$$
[\boldsymbol{a}]_{\times}=\left[\begin{array}{ccc}
0 & -a(3) & a(2) \\
a(3) & 0 & -a(1) \\
-a(2) & a(1) & 0
\end{array}\right] \text {. }
$$

$\mathbf{R}_{\theta, \boldsymbol{V}}$ describes a rotation by the angle $\theta=\operatorname{atan} 2\left(\left\|\boldsymbol{W}_{1} \times \boldsymbol{W}_{2}\right\|, \boldsymbol{W}_{1} \cdot \boldsymbol{W}_{2}\right)$ around the unit axis $\boldsymbol{V}=\boldsymbol{W}_{1} \times \boldsymbol{W}_{2} / \sin \theta$, with atan2 being the four-quadrant inverse tangent function.

Given $\theta$ constant (the angle has to be maintained), the variation of the rotation matrix with respect to its axis times $\boldsymbol{W}_{1}$ is given by

$$
\delta \mathbf{R}_{\theta, \boldsymbol{V}} \boldsymbol{W}_{1}=\left(-\sin \theta\left[\boldsymbol{W}_{1}\right]_{\times}+(1-\cos \theta) \boldsymbol{V} \boldsymbol{W}_{1}^{T}\right) \delta \boldsymbol{V}
$$

Replacing $\delta \boldsymbol{V}$ in terms of $\delta \boldsymbol{W}_{1}$ and $\delta \boldsymbol{W}_{2}$, Eq. (A.3) can be recasted as

$$
\delta \mathbf{R}_{\theta, \boldsymbol{V}} \boldsymbol{W}_{1}=-\left[\boldsymbol{W}_{1}\right]_{\times}^{2} \delta \boldsymbol{W}_{2}+\left(\left[\boldsymbol{W}_{1}\right]_{\times}\left[\boldsymbol{W}_{2}\right]_{\times}-(1-\cos \theta) \boldsymbol{V} \boldsymbol{V}^{T}\right) \delta \boldsymbol{W}_{1} .
$$

Finally, the variation of Eq. (A.1) reads

$$
\left(\mathbf{I}+\left[\boldsymbol{W}_{1}\right]_{\times}^{2}\right) \delta \boldsymbol{W}_{2}=\left(\mathbf{R}_{\theta, \boldsymbol{V}}+\left[\boldsymbol{W}_{1}\right]_{\times}\left[\boldsymbol{W}_{2}\right]_{\times}-(1-\cos \theta) \boldsymbol{V} \boldsymbol{V}^{T}\right) \delta \boldsymbol{W}_{1} .
$$

Noting that the determinant of the left-hand side of Eq. (A.5) is $\left|\mathbf{I}+\left[\boldsymbol{W}_{1}\right]_{\times}^{2}\right|$ $=\left(\left\|\boldsymbol{W}_{1}\right\|^{2}-1\right)^{2}=0, \delta \boldsymbol{W}_{1}$ can be expressed in terms of $\delta \boldsymbol{W}_{2}$, but the problem (A.5) is ill-posed. 


\section{References}

[1] C. Claeys, E. Deckers, B. Pluymers, and W. Desmet, "A lightweight vibro-acoustic metamaterial demonstrator: Numerical and experimental investigation," Mechanical Systems and Signal Processing, vol. 70-71, pp. 853-880, 2016.

[2] V. Dorodnitsyn and B. Van Damme, "Two-dimensional fluid-filled closed-cell cellular solid as an acoustic metamaterial with negative index," Physical Review B, vol. 93, p. 134302, apr 2016.

[3] P. E. Mix, Introduction to Nondestructive Testing: A Training Guide. John Wiley \& Sons, 2005.

[4] L. Brillouin, Wave propagation in periodic structures; electric filters and crystal lattices. Courier Dover Publications, 1946.

[5] D. J. Mead, "A new method of analyzing wave propagation in periodic structures; Applications to periodic Timoshenko beams and stiffened plates," Journal of Sound and Vibration, vol. 104, pp. 9-27, jan 1986.

[6] R. S. Langley, "A note on the force boundary conditions for twodimensional periodic structures with corner freedoms," Journal of Sound and Vibration, vol. 167, pp. 377-381, oct 1993.

[7] M. I. Hussein, M. J. Leamy, and M. Ruzzene, "Dynamics of phononic materials and structures: Historical origins, recent progress, and future outlook," Applied Mechanics Reviews, vol. 66, p. 040802, may 2014.

[8] J. F. Doyle, Wave Propagation in Structures-Spectral Analysis Using Fast Discrete Fourier Transforms. Springer Verlag, 1997.

[9] F. Maurin, L. Dedè, and A. Spadoni, "Isogeometric rotation-free analysis of planar extensible-elastica for static and dynamic applications," Nonlinear Dynamics, vol. 81, pp. 77-96, mar 2015.

[10] J. A. Cottrell, T. J. R. Hughes, and Y. Bazilevs, Isogeometric Analysis Toward Intergration of CAD and FEA. John Wiley \& Sons, 2009.

[11] S. B. Raknes, X. Deng, Y. Bazilevs, D. J. Benson, K. M. Mathisen, and T. Kvamsdal, "Isogeometric rotation-free bending-stabilized cables: 
Statics, dynamics, bending strips and coupling with shells," Computer Methods in Applied Mechanics and Engineering, vol. 263, pp. 127-143, aug 2013.

[12] L. Greco and M. Cuomo, "B-Spline interpolation of Kirchhoff-Love space rods," Computer Methods in Applied Mechanics and Engineering, vol. 256, no. 0, pp. 251-269, 2013.

[13] A. M. Bauer, M. Breitenberger, B. Philipp, R. Wüchner, and K. U. Bletzinger, "Nonlinear isogeometric spatial Bernoulli beam," Computer Methods in Applied Mechanics and Engineering, vol. 303, pp. 101-127, may 2016.

[14] J. Kiendl, K. U. Bletzinger, J. Linhard, and R. Wüchner, "Isogeometric shell analysis with Kirchhoff-Love elements," Computer Methods in Applied Mechanics and Engineering, vol. 198, no. 49-52, pp. 3902-3914, 2009.

[15] J. Kiendl, M. C. Hsu, M. C. H. Wu, and A. Reali, "Isogeometric Kirchhoff-Love shell formulations for general hyperelastic materials," Computer Methods in Applied Mechanics and Engineering, vol. 291, pp. 280-303, 2015.

[16] N. Nguyen-Thanh, N. Valizadeh, M. N. Nguyen, H. Nguyen-Xuan, X. Zhuang, P. Areias, G. Zi, Y. Bazilevs, L. De Lorenzis, and T. Rabczuk, "An extended isogeometric thin shell analysis based on Kirchhoff-Love theory," Computer Methods in Applied Mechanics and Engineering, vol. 284, pp. 265-291, feb 2015.

[17] T. X. Duong, F. Roohbakhshan, and R. A. Sauer, "A new rotation-free isogeometric thin shell formulation and a corresponding continuity constraint for patch boundaries," Computer Methods in Applied Mechanics and Engineering, apr 2016.

[18] P. Fischer, M. Klassen, J. Mergheim, P. Steinmann, and R. Müller, "Isogeometric analysis of 2D gradient elasticity," Computational Mechanics, vol. 47, pp. 325-334, oct 2010.

[19] F. Auricchio, L. B. da Veiga, A. Buffa, C. Lovadina, A. Reali, and G. Sangalli, "A fully "locking-free" isogeometric approach for plane 
linear elasticity problems: A stream function formulation," Computer Methods in Applied Mechanics and Engineering, vol. 197, pp. 160-172, dec 2007.

[20] H. Gómez, V. M. Calo, Y. Bazilevs, and T. J. R. Hughes, "Isogeometric analysis of the CahnHilliard phase-field model," Computer Methods in Applied Mechanics and Engineering, vol. 197, pp. 4333-4352, sep 2008.

[21] A. Bartezzaghi, L. Dedè, and A. Quarteroni, "Isogeometric Analysis of high order Partial Differential Equations on surfaces," Computer Methods in Applied Mechanics and Engineering, vol. 295, pp. 446-469, oct 2015 .

[22] A. Tagliabue, L. Dedè, and A. Quarteroni, "Isogeometric Analysis and error estimates for high order partial differential equations in fluid dynamics," Computers $\& 3$ Fluids, vol. 102, pp. 277-303, oct 2014.

[23] J. A. Cottrell, T. J. R. Hughes, and A. Reali, "Studies of refinement and continuity in isogeometric structural analysis," Computer Methods in Applied Mechanics and Engineering, vol. 196, no. 41, pp. 4160-4183, 2007.

[24] L. Coox, F. Greco, O. Atak, D. Vandepitte, and W. Desmet, "A robust patch coupling method for NURBS-based isogeometric analysis of non-conforming multipatch surfaces," Computer Methods in Applied Mechanics and Engineering, In press, 2016.

[25] L. Coox, O. Atak, D. Vandepitte, and W. Desmet, "An isogeometric indirect boundary element method for solving acoustic problems in openboundary domains," Computer Methods in Applied Mechanics and Engineering, In press, 2016.

[26] W. Dornisch, G. Vitucci, and S. Klinkel, "The weak substitution method - an application of the mortar method for patch coupling in NURBSbased isogeometric analysis," International Journal for Numerical Methods in Engineering, vol. 103, pp. 205-234, jul 2015.

[27] W. Dornisch, J. Stöckler, and R. Müller, "Dual and approximate dual basis functions for B-splines and NURBS Comparison and application for an efficient coupling of patches with the isogeometric mortar 
method," Computer Methods in Applied Mechanics and Engineering, 2016.

[28] V. P. Nguyen, P. Kerfriden, M. Brino, S. P. A. Bordas, and E. Bonisoli, "Nitsche's method for two and three dimensional NURBS patch coupling," Computational Mechanics, vol. 53, pp. 1163-1182, jun 2014.

[29] E. Brivadis, A. Buffa, B. Wohlmuth, and L. Wunderlich, "Isogeometric mortar methods," Computer Methods in Applied Mechanics and Engineering, vol. 284, pp. 292-319, 2015.

[30] K. Sommerwerk, M. Woidt, M. C. Haupt, and P. Horst, "ReissnerMindlin shell implementation and energy conserving isogeometric multipatch coupling," International Journal for Numerical Methods in Engineering, 2016.

[31] A. Apostolatos, R. Schmidt, R. Wüchner, and K.-U. Bletzinger, "A Nitsche-type formulation and comparison of the most common domain decomposition methods in isogeometric analysis," International Journal for Numerical Methods in Engineering, vol. 97, pp. 473-504, feb 2014.

[32] J. Kiendl, Y. Bazilevs, M. C. Hsu, R. Wüchner, and K. U. Bletzinger, "The bending strip method for isogeometric analysis of Kirchhoff-Love shell structures comprised of multiple patches," Computer Methods in Applied Mechanics and Engineering, vol. 199, no. 37-40, pp. 2403-2416, 2010.

[33] D. J. Benson, S. Hartmann, Y. Bazilevs, M. C. Hsu, and T. J. R. Hughes, "Blended isogeometric shells," Computer Methods in Applied Mechanics and Engineering, vol. 255, no. 0, pp. 133-146, 2013.

[34] L. Greco and M. Cuomo, "An implicit G1 multi patch B-spline interpolation for Kirchhoff-Love space rod," Computer Methods in Applied Mechanics and Engineering, vol. 269, no. 0, pp. 173-197, 2014.

[35] L. Greco and M. Cuomo, "An isogeometric implicit G1 mixed finite element for Kirchhoff space rods," Computer Methods in Applied Mechanics and Engineering, vol. 298, pp. 325-349, 2016. 
[36] W. Dornisch, S. Klinkel, and B. Simeon, "Isogeometric Reissner-Mindlin shell analysis with exactly calculated director vectors," Computer Methods in Applied Mechanics and Engineering, vol. 253, pp. 491-504, 2013.

[37] W. Dornisch and S. Klinkel, "Treatment of Reissner-Mindlin shells with kinks without the need for drilling rotation stabilization in an isogeometric framework," Computer Methods in Applied Mechanics and Engineering, vol. 276, pp. 35-66, 2014.

[38] D. Koks, Explorations in mathematical physics: The concepts behind an elegant language. Springer New York, 2006.

[39] M. Ruzzene, F. Scarpa, and F. Soranna, "Wave beaming effects in two-dimensional cellular structures," Smart Materials and Structures, vol. 12, pp. 363-372, jun 2003.

[40] A. S. Phani, J. Woodhouse, and N. A. Fleck, "Wave propagation in twodimensional periodic lattices.," The Journal of the Acoustical Society of America, vol. 119, no. 4, pp. 1995-2005, 2006.

[41] M. J. Leamy, "Exact wave-based Bloch analysis procedure for investigating wave propagation in two-dimensional periodic lattices," Journal of Sound and Vibration, vol. 331, pp. 1580-1596, mar 2012.

[42] V. Dorodnitsyn and A. Spadoni, "Elastodynamics of a Two- Dimensional Square Lattice With Entrained Fluid - Part I: Comparison With Biot's Theory," Journal of Vibration and Acoustics, vol. 136, p. 021024, feb 2014.

[43] G. Carta, M. Brun, A. B. Movchan, N. V. Movchan, and I. S. Jones, "Dispersion properties of vortex-type monatomic lattices," International Journal of Solids and Structures, vol. 51, pp. 2213-2225, jun 2014.

[44] B. R. Mace, D. Duhamel, M. J. Brennan, and L. Hinke, "Finite element prediction of wave motion in structural waveguides.," The Journal of the Acoustical Society of America, vol. 117, pp. 2835-2843, apr 2005.

[45] M. Collet, M. Ouisse, M. Ruzzene, and M. N. Ichchou, "Floquet-Bloch decomposition for the computation of dispersion of two-dimensional periodic, damped mechanical systems," International Journal of Solids and Structures, vol. 48, pp. 2837-2848, oct 2011. 
[46] F. Farzbod and M. J. Leamy, "Analysis of Bloch's Method and the Propagation Technique in Periodic Structures," Journal of Vibration and Acoustics, vol. 133, no. 3, p. 031010, 2011.

[47] L. Piegl and W. Tiller, The NURBS Book. Springer Science \& Business Media, 1996.

[48] J. Kiendl, Isogeometric Analysis and Shape Optimal Design of Shell Structures. PhD thesis, Technische Universität München, 2011.

[49] F. Scarpa, M. Ouisse, M. Collet, and K. Saito, "Kirigami Auxetic Pyramidal Core: Mechanical Properties and Wave Propagation Analysis in Damped Lattice," Journal of Vibration and Acoustics, vol. 135, p. 041001, jun 2013.

[50] W. X. Zhong and F. W. Williams, "On the direct solution of wave propagation for repetitive structures," Journal of Sound and Vibration, vol. 181, pp. 485-501, mar 1995.

[51] F. Auricchio, L. B. Da Veiga, T. J. R. Hughes, A. Reali, and G. Sangalli, "Isogeometric Collocation Methods," Mathematical Models and Methods in Applied Sciences, vol. 20, pp. 2075-2107, nov 2010.

[52] C. de Boor, A practical guide to splines. Springer-Verlag, 1979.

[53] T. J. R. Hughes, J. A. Evans, and A. Reali, "Finite element and NURBS approximations of eigenvalue, boundary-value, and initial-value problems," Computer Methods in Applied Mechanics and Engineering, vol. 272, pp. 290-320, apr 2014.

[54] L. Dedè and A. Quarteroni, "Isogeometric Analysis for second order Partial Differential Equations on surfaces," Computer Methods in Applied Mechanics and Engineering, vol. 284, pp. 807-834, feb 2015.

[55] J. A. Cottrell, A. Reali, Y. Bazilevs, and T. J. R. Hughes, "Isogeometric analysis of structural vibrations," Computer Methods in Applied Mechanics and Engineering, vol. 195, pp. 5257-5296, aug 2006.

[56] D. Wang, W. Liu, and H. Zhang, "Superconvergent isogeometric free vibration analysis of Euler-Bernoulli beams and Kirchhoff plates with new higher order mass matrices," Computer Methods in Applied Mechanics and Engineering, vol. 286, pp. 230-267, apr 2015. 
[57] F. Maurin, "Bloch theorem with revised boundary conditions applied to glide and screw symmetric, quasi-one-dimensional structures," Wave Motion, vol. 61, pp. 20-39, nov 2016.

[58] A. P. Korte, E. L. Starostin, and G. H. M. van der Heijden, "Triangular buckling patterns of twisted inextensible strips," Proceedings of the Royal Society A: Mathematical, Physical and Engineering Sciences, vol. 467, pp. 285-303, jun 2010.

[59] J. Chopin, V. Démery, and B. Davidovitch, "Roadmap to the Morphological Instabilities of a Stretched Twisted Ribbon," Journal of Elasticity, vol. 119, pp. 137-189, sep 2014.

[60] F. Maurin, "Solitary waves in longitudinally wrinkled and creased helicoids," Submitted for publication, 2016.

[61] F. Maurin, C. Claeys, L. Van Belle, and W. Desmet, "Bloch theorem with revised boundary conditions applied to glide, screw and rotational symmetric structures," Submitted for publication, 2016.

[62] G. Trainiti, J. J. Rimoli, and M. Ruzzene, "Wave propagation in periodically undulated beams and plates," International Journal of Solids and Structures, vol. 75-76, pp. 260-276, sep 2015.

[63] F. Higuchi, S. Gofuku, T. Maekawa, H. Mukundan, and N. M. Patrikalakis, "Approximation of involute curves for CAD-system processing," Engineering with Computers, vol. 23, pp. 207-214, apr 2007.

[64] D. Smith, "Recent Patents on Expanded Metal," Recent Patents on Materials Science, vol. 2, oct 2016. 